1 Article

\title{
2 Evidence of a causal relationship between genetic tendency to 3 gain muscle mass and uterine leiomyomata
}

4 Eeva Sliz ${ }^{1,2}$, Jaakko Tyrmi ${ }^{1,2}$, Nilufer Rahmioglu ${ }^{6,7}$, Krina T. Zondervan ${ }^{6,7}$, Christian M.

5 Becker $^{6}$, FinnGen, Outi Uimari ${ }^{3,4,5}$, Johannes Kettunen ${ }^{1,2} *$

$6{ }^{1}$ Center for Life Course Health Research, Faculty of Medicine, University of Oulu, Finland

$7 \quad{ }^{2}$ Biocenter Oulu, Oulu, Finland

$8 \quad{ }^{3}$ Department of Obstetrics and Gynecology, Oulu University Hospital, Oulu, Finland

$9 \quad{ }^{4}$ PEDEGO Research Unit, University of Oulu and Oulu University Hospital, Oulu, Finland

$10{ }^{5}$ Medical Research Center Oulu, University of Oulu and Oulu University Hospital, Oulu,

11 Finland

$12{ }^{6}$ Oxford Endometriosis CaRe Centre, Nuffield Department of Women's and Reproductive

13 Health, University of Oxford, UK

$14 \quad{ }^{7}$ Wellcome Centre for Human Genetics, University of Oxford, UK

$15 *$ Equal contribution of the last authors

16 Address for correspondence:

17 Eeva Sliz

18 Center For Life Course Health Research

19 Faculty of Medicine

20 Aapistie 5A

21 P.O.Box 5000, 90014 University of Oulu

22 Oulu, Finland

23 phone: +358503054865

24 e-mail: eeva.sliz@oulu.fi

25 Word count - Introductory paragraph: 150

26 Word count - Manuscript main body: 1627

27 Figures: 4

28 Tables: 1

29 Supplementary figures: 63

30 Supplementary tables: 9

31 References: 68

32 Key words: Uterine leiomyoma, GWAS, smooth muscle, muscle mass

33 Summary statistics will be made available through the NHGRI-EBI GWAS Catalog with

34 GCSTXxxxxxx upon publication. 
Uterine leiomyomata (UL) are the most common benign tumours of the female genital tract with an estimated lifetime incidence of up to $70 \%{ }^{1}$. To date, 7 genome-wide association studies (GWASs) have identified 35 loci predisposing to UL. To improve the understanding of the underlying genetic pathways, we conducted the largest genetic association study of UL to date in 426,558 European women from FinnGen and a previously published UL meta-GWAS ${ }^{2}$. We identified 36 novel and replicated 31 previously reported loci. Annotations of the potential candidate genes suggest involvement of smooth muscle cell (SMC) differentiation and/or proliferationregulating genes in modulating UL risk. Our results further advocate that genetic predisposition to increased fat-free mass may be causally related to UL risk, underscoring the involvement of altered muscle tissue biology in UL pathophysiology. Overall, our findings provide novel insights into genetic risk factors related to UL, which may aid in developing novel treatment strategies.

UL are hormone-driven benign neoplasms of the uterus composed mostly of SMC and fibroblasts with a profound component of extracellular matrix (ECM). UL are present in single or multiple numbers, with size ranging from millimeters to $20 \mathrm{~cm}$ or more in diameter $^{3}$. In $25-50 \%$ of women with ULs, the enlarged and deformed uterus can cause symptoms such as heavy or prolonged menstrual bleeding resulting in anemia, reduced fertility, and pregnancy complications ${ }^{4}$. Major risk factors for UL are family history, African ancestry, increasing age up to menopause, and nulliparity ${ }^{5}$. Also, metabolic factors, including hypertension and increased body mass index (BMI), have been reported in association with UL susceptibility ${ }^{6}$. Familial aggregation, twin studies, and disparity in prevalence between different ethnic groups suggest that genetic factors modulate UL risk ${ }^{7-10}$.

Until recently, the focus in the genetics of UL has been on somatic rearrangements, and key driver variations in MED12, FH, HMGA2 and COLAA6-COLAA5 have been reported ${ }^{11}$. To add understanding to the heritable genetic underpinnings, we conducted two sets of fixedeffect, inverse-variance weighted meta-analyses with data from FinnGen (18,060 cases and 105,519 female controls) and 1) a previously published UL meta-GWAS ${ }^{2}$ totaling 53,534 cases and 373,024 female controls in 'META-1' and 2) the UK Biobank totaling 33,244 cases and 311,271 controls in 'META-2'. META-1 was restricted to publicly available 10,000 variants from the previous meta-GWAS ${ }^{2}$, wheras the META-2 included 10,693,588 variants across the genome. In META-1, we identified 63 loci associating with UL at $p<5 \times 10^{-8}$ (Figure 1, Table S1); of these, 32 were novel (Table 1). In META-2, we identified 51 loci out of which 4 had not been associated with UL risk in prior GWASs or in META-1 (Figure 1, Table 1, Table S2). Regional association plots of the novel loci are presented in Figures S1-S36. There was no evidence of inflation in the test statistics (Figure S37). LD score (LDSC) regression-derived SNP-based heritability was estimated to be 0.099 (standard error $=0.01$ ) on the liability scale. Characterization of the genome-wide results of META-2 suggested that the key UL associated variants were mostly intronic (Figure 2A). Genes associated with UL risk (Figure 2B) were significantly enriched on pathways related to gonad development, positive regulation of growth, and cellular senescence (Figure 2C) and they were mostly expressed in uterus, cervix, and fallopian tube (Figure 2D). In two of the novel loci, we found evidence that altered gene expression mediates UL association (Figures S38S40).

Previous GWAS findings ${ }^{2,12-17}$ have indicated that genetic factors altering pathways involved in estrogen signalling, Wnt signalling, transforming growth factor (TGF)- $\beta$ signalling, and cell cycle progression are associated with UL risk ${ }^{18}$. The novel loci identified in this study further underscore the involvement of pathways regulating SMC proliferation in modulation 
84 progesterone increase the secretion of Wnt ligands from myometrial or leiomyoma SMC, hormones also influence the production of ECM via signalling through the TGF- $\beta$ family of ligands and receptors that are highly expressed in multiple fibrotic conditions ${ }^{20-26}$ and contribute to the fibrotic phenotype seen in $\mathrm{UL}^{24,25,27-29}$. We identified multiple loci with potential candidate genes functioning in one or more of these pathways, and, in the following, we describe some of our key findings with the focus on loci involved in regulation of SMC proliferation.

A central finding is the novel association at $17 \mathrm{p} 12$ harbouring myocardin (MYOCD; Figure S27). Myocardin is a transcription factor expressed in smooth muscle tissues, including most prominently arteries and colon, but also uterus (Figure S41), and it is required for SMC differentiation $^{30}$. The expression of myocardin has been shown to be downregulated in UL tissue compared with normal myometrium ${ }^{31}$. Also, it has been proposed that the loss of myocardin function may be a key factor in driving SMC proliferation in $\mathrm{UL}^{31}$; however, there are no reported GWAS associations implicating myocardin until now. The lead variants near MYOCD are intergenic variants with no strong evidence of altered regulatory consequences (Table S3) and, thus, a possible association-driving mechanism remains inconclusive. We identified another myocardin-related novel UL risk association near 'myocardin-induced smooth muscle cell lncRNA, inducer of differentiation' (MYOSLID), a transcriptional target of myocardin ${ }^{32}$ (Figure S6). RegulomeDB ${ }^{33}$ annotation provided robust evidence that the association lead variant is a regulatory variant (Table S4) altering binding of multiple transcription factors (Table S5), including Fos proto-oncogene (FOS) that has been shown to be downregulated in $\mathrm{UL}^{34}$. To add yet another example of a myocardin-related UL risk loci, a well-established UL risk association at 22q13.1,12,16,35,36 locates near 'myocardin related transcription factor A' (MRTFA; also known as MKL1), a gene interacting with myocardin ${ }^{37}$. Also, expression of MRTFA/MKL1 has been shown to be downregulated in UL-related deletions at $22 \mathrm{q}^{38}$.

111 Others have suggested that loss of myocardin function may account for the differentiation 112 defects of human leiomyosarcoma cells during malignant transformation ${ }^{39}$ : downregulation of myocardin resulted in lower expression of cyclin dependent kinase inhibitor $1 \mathrm{~A}$ (CDKN1A; also known as p21), a mediator of cell cycle G1 phase arrest, which facilitated cell cycle progression. We identified a novel UL association at $6 \mathrm{p} 21.2$ near CDKN1A (Figure $\mathrm{S} 13)$; the association lead variants locate on intergenic region with possible regulatory consequences (Table S6). Previous evidence suggests that CDKN1A is among the genes, the expression of which correlates with UL size ${ }^{40}$. Another novel UL association at 20q13.31 harbours RNA binding motif protein 38 (RBM38; Figure S29) that specifically binds to and regulates the stability of $C D K N 1 A$ transcripts ${ }^{37}$. In this locus, the UL risk-increasing rs13039273-C associates with lower RBM38 expression in ovary $\left(p=8.7 \times 10^{-6}\right)$ (Table S7, Figure S42; eQTL in uterus $p=2.2 \times 10^{-3}$ ). Relevant to UL, a variant in the same locus (rs760640162) has been previously associated with 'pregnancy examination and test' $\left(p=5.97 \times 10^{-15}\right)$ in the UK Biobank ${ }^{41,42}$. Interestingly, estrogen receptor (ER) $\alpha$ has been shown to inhibit expression of myocardin ${ }^{31}$, suggesting that the ability of myocardin-CDKN1Asignalling to inhibit cell cycle progression may be impaired in tissues enriched with ER $\alpha$. Taken together our findings and previous evidence, it seems highly probable that downregulation of myocardin-CDKN1A signalling increases the risk of UL.

We used an automated LDSC regression pipeline ${ }^{43}$ to evaluate the genetic correlations $\left(r_{\mathrm{g}}\right)$ of UL with other traits (Figure 2; Table S8). The most robustly correlated traits were seen for other gynecological traits, such as bilateral oophorectomy $\left(\mathrm{r}_{\mathrm{g}}=0.74, p=1.05 \times 10^{-25}\right)$, hysterectomy $\left(r_{\mathrm{g}}=0.62, p=3.24 \times 10^{-19}\right)$, age at menarche $\left(r_{\mathrm{g}}=-0.18, p=1.40 \times 10^{-9}\right)$, excessive 
133 frequent and irregular menstruation $\left(\mathrm{r}_{\mathrm{g}}=0.51, p=5.66 \times 10^{-7}\right)$, having had menopause $\left(\mathrm{r}_{\mathrm{g}}=-0.33\right.$, $\left.134 p=2.31 \times 10^{-5}\right)$, and usage of hormonal replacement therapy $\left(\mathrm{r}_{\mathrm{g}}=0.20, \mathrm{p}=5.54 \times 10^{-5}\right)$. Strong 135 correlations were also seen between UL and anthropometric and metabolic measures, 136 including whole body fat-free mass $\left(\mathrm{r}_{\mathrm{g}}=0.11, p=4.21 \times 10^{-5}\right)$, high blood pressure $\left(\mathrm{r}_{\mathrm{g}}=0.15\right.$, $\left.137 p=4.58 \times 10^{-6}\right)$, and serum triglyceride level $\left(\mathrm{r}_{\mathrm{g}}=0.16, p=4.80 \times 10^{-5}\right)$. Genetic susceptibility for 138 UL was also correlated with genetic susceptibility for multiple psychiatric or mood-related 139 traits, including depressive symptoms $\left(\mathrm{r}_{\mathrm{g}}=0.20, p=3.31 \times 10^{-5}\right)$ or neuroticism score $\left(\mathrm{r}_{\mathrm{g}}=0.14\right.$, $\left.140 p=3.88 \times 10^{-5}\right)$.

141 To estimate the causal relationships between UL and the key traits showing significant $r_{g}$ 142 with UL, we applied two-sample Mendelian randomization. Our results suggest that genetic 143 tendency to accumulate fat-free mass may be causally linked to higher risk of UL $144\left(p=2.37 \times 10^{-3}\right.$; Figure 3; Table S9). In line with this, the higher impedance of whole body (i.e., 145 a bioelectrical measure used for estimating body composition; lower muscle mass leads to 146 higher impedance) was causally associated with a lower risk of UL $\left(p=1.05 \times 10^{-3}\right)$. When 147 considering the null causal effect of whole-body fat mass on UL risk $(p=0.46)$, it seems 148 apparent that the nominally significant $\left(p=8.40 \times 10^{-3}\right)$ causal effect of BMI on UL in the 149 present study and the previously reported associations of BMI with UL risk ${ }^{44-46}$ arise from the 150 increased lean body mass rather than fat mass. Although we did not observe horizontal pleiotropy, the causal estimates were heterogenic for many key traits (Table S9), suggesting that all the underlying genetic pathways may not function in a consistent manner - thus, these results need to be interpreted with caution. However, in the leave-one-out sensitivity analyses all causal estimates were consistently positive (higher fat-free mass was causally associated with higher risk of UL) or negative (higher impedance was causally associated with lower risk of UL) suggesting that there is no single variant driving the causal associations (Figures S50-S63). Regarding most of the gynecological traits, the causal direction with UL was not clear (Table S9), suggesting that the shared molecular factors predispose to multiple gynecological complications simultaneously instead of one being causal to another. Of note, we replicated the previously reported finding of genetic predisposition to UL being causal to excessive menstrual bleeding ${ }^{2}\left(p=1.07 \times 10^{-4}\right)$.

162 The discovered causal relationship between genetic tendency to accumulate fat-free mass and 163 UL risk provides a novel perspective on UL-related pathophysiology. UL are considered estrogen-dependent, and UL have higher ER $\alpha$ expression compared with normal uterine myometria $^{47,48}$. ERs are expressed in a variety of tissues, including all musculoskeletal tissues $^{49}$. In females, muscle mass and strength are closely coupled with estrogen status: girls begin to gain muscle mass after the onset of puberty ${ }^{50}$ whereas in older age during perimenopausal and postmenopausal periods, muscle strength declines considerably ${ }^{51,52}$. If estrogen enhances muscle growth ${ }^{53}$, the observed causal relationship between fat-free mass and UL risk could arise secondary to high estrogen contributing to the muscle growth. Many of the newly identified candidate genes, however, directly regulate cell proliferation - thus, it remains possible that the estrogen-rich environment, due to sexual maturity, may trigger excess SMC growth resulting in ULs in women who are genetically susceptible to build up muscle.

175 Currently, the only essentially curative treatment for UL is hysterectomy, which underscores 176 the high demand for the development of alternative effective therapies. Our findings double 177 the number of known UL risk loci and, thus, the herein presented results provide several potential targets for translational research to develop pharmacologic interventions for UL. Therapies targeted at myocardin-CDKN1A signalling or, considering the causal evidence, 180 other factors regulating muscle growth may hold the greatest potential. 
1 Table 1. Novel UL risk loci.

2 The table reports novel distinct loci (more than $1 \mathrm{Mb}$ apart) containing at least one variant identified to be associated with $\mathrm{UL}$ at $p<5 \times 10^{-8}$ in a meta-analysis of

3 53,534 UL cases and 373,024 female controls from FinnGen and a previously published meta-GWAS of UL ${ }^{2}$ (META-1) and in a meta-analysis of 33,244 UL

4 cases and 311,271 female controls from FinnGen and the UK Biobank (META-2).

\begin{tabular}{|c|c|c|c|c|c|c|c|c|c|c|}
\hline Locus & Chr:Pos (hg38) & Nearest gene(s) & Candidate gene(s) & rsID & EA & EAF & OR $(95 \% \mathrm{CI})$ & P-value & HetPVal & FIN enr. \\
\hline \multicolumn{11}{|c|}{ Novel loci in META-1 } \\
\hline $1 \mathrm{q} 43$ & $1: 241860596$ & EXO1 & EXO1, FH & rs4149909 & G & 0.03 & $1.13(1.08-1.18)$ & $1.16 \mathrm{E}-08$ & 0.265 & 1.03 \\
\hline $1 \mathrm{q} 44$ & $1: 244151650$ & ZBTB18, Clorf100 & ZВTB18, АKT3 & rs2183478 & G & 0.18 & $1.07(1.05-1.09)$ & $1.75 \mathrm{E}-11$ & 0.774 & 1.57 \\
\hline $1 \mathrm{q} 44$ & $1: 248897507$ & $P G B D 2$ & ZNF692 & rs4335411 & A & 0.76 & $1.06(1.04-1.08)$ & $4.12 \mathrm{E}-10$ & 0.376 & 0.98 \\
\hline $2 \mathrm{p} 14$ & $2: 66863235$ & MEISI & MEISI & rs 17631680 & $\mathrm{C}$ & 0.10 & $0.93(0.91-0.95)$ & $1.93 \mathrm{E}-10$ & 0.127 & 0.96 \\
\hline $2 q 11.2$ & 2:99454113 & REVI & $R E V 1$ & rs13392042 & G & 0.58 & $1.05(1.03-1.06)$ & $4.21 \mathrm{E}-11$ & 0.217 & 1.21 \\
\hline $2 q 33.3$ & $2: 207258660$ & MYOSLID, KLF7 & MYOSLID & rs10804157 & $\mathrm{C}$ & 0.44 & $1.04(1.03-1.05)$ & $1.04 \mathrm{E}-08$ & 0.143 & 0.87 \\
\hline $2 q 37.3$ & $2: 241720139$ & ING5 & ING5 & rs34766121 & $\mathrm{T}$ & 0.24 & $1.06(1.04-1.08)$ & $5.02 \mathrm{E}-10$ & 0.278 & 1.03 \\
\hline $3 \mathrm{p} 26.1$ & 3:4674530 & ITPRI & ITPRI & rs3804984 & $\mathrm{C}$ & 0.38 & $0.95(0.93-0.96)$ & $8.06 \mathrm{E}-15$ & 0.021 & 1.42 \\
\hline $3 \mathrm{p} 24.2$ & $3: 24213259$ & THRB & THRB & rs1010961 & A & 0.45 & $1.04(1.03-1.06)$ & $1.64 \mathrm{E}-10$ & 0.109 & 0.98 \\
\hline $3 q 27.2$ & $3: 185807411$ & $I G F 2 B P 2$ & $I G F 2 B P 2$ & rs13060777 & G & 0.26 & $1.05(1.04-1.07)$ & $1.14 \mathrm{E}-11$ & 0.595 & 1.10 \\
\hline $5 q 31.1$ & $5: 133099880$ & HSPA4 & HSPA4 & rs4367292 & $\mathrm{T}$ & 0.27 & $0.96(0.94-0.97)$ & $2.49 \mathrm{E}-08$ & 0.882 & 1.15 \\
\hline 6p21.2 & $6: 36653670$ & CDKNIA & CDKNIA & rs10456443 & A & 0.20 & $0.95(0.93-0.96)$ & $2.67 \mathrm{E}-10$ & 0.039 & 0.91 \\
\hline $6 \mathrm{q} 21$ & 6:109054915 & SESNI & SESN1 & rs11153158 & $\mathrm{C}$ & 0.13 & $0.93(0.92-0.95)$ & $1.05 \mathrm{E}-10$ & 0.243 & 1.19 \\
\hline $7 \mathrm{p} 14.3$ & 7:33008785 & FКВP9, NT5C3A & NT5C3A, BBS9 & rs 4723230 & $\mathrm{~T}$ & 0.80 & $1.05(1.03-1.07)$ & $4.68 \mathrm{E}-08$ & 0.946 & 1.03 \\
\hline $7 \mathrm{q} 31.31$ & $7: 121132432$ & CPED1 & WNT16 & rs12706314 & A & 0.53 & $1.04(1.03-1.06)$ & $2.69 \mathrm{E}-10$ & 0.777 & 0.89 \\
\hline $7 \mathrm{q} 32.3$ & 7:130935964 & LINC-PINT & LINC-PINT & rs35908158 & $\mathrm{C}$ & 0.08 & $1.08(1.05-1.10)$ & $1.60 \mathrm{E}-08$ & 0.883 & 1.41 \\
\hline $8 \mathrm{p} 12$ & $8: 30452819$ & $R B P M S$ & $R B P M S$ & rs13275869 & $\mathrm{C}$ & 0.49 & $0.96(0.95-0.97)$ & 8.64E-09 & 0.520 & 0.98 \\
\hline $8 \mathrm{q} 24.21$ & $8: 128506035$ & LINC00824 & LINCO0824 & rs1516980 & $\mathrm{C}$ & 0.25 & $0.96(0.94-0.97)$ & $2.72 \mathrm{E}-08$ & 0.987 & 0.76 \\
\hline $9 \mathrm{q} 22.2$ & $9: 89639982$ & $G A D D 45 G$ & $G A D D 45 G$ & $\mathrm{rs} 28508285$ & G & 0.09 & $1.07(1.04-1.09)$ & $1.49 \mathrm{E}-08$ & 0.362 & 1.05 \\
\hline $10 \mathrm{p} 12.31$ & $10: 21517903$ & SKIDAl & DNAJCl & rs946711 & $\mathrm{C}$ & 0.33 & $1.05(1.03-1.06)$ & $2.96 \mathrm{E}-10$ & 0.460 & 0.96 \\
\hline $10 \mathrm{q} 23.31$ & 10:88331783 & $R N L S$ & $R N L S$ & rs1426619 & $\mathrm{T}$ & 0.45 & $1.04(1.03-1.06)$ & 4.92E-09 & 0.653 & 1.09 \\
\hline $15 \mathrm{q} 23$ & $15: 67922458$ & SKORI & PIAS1 & rs12148374 & $\mathrm{C}$ & 0.44 & $0.96(0.95-0.97)$ & $1.37 \mathrm{E}-09$ & 0.832 & 1.10 \\
\hline $16 q 12.1$ & $16: 50059327$ & HEATR3 & $B R D 7$ & rs12599260 & A & 0.73 & $1.05(1.04-1.07)$ & $1.09 \mathrm{E}-11$ & 0.002 & 1.08 \\
\hline 16q12.1 & $16: 51447685$ & AC007344.1 & AC007344.1 & rs66998222 & A & 0.19 & $0.94(0.93-0.96)$ & $7.53 \mathrm{E}-12$ & 0.345 & 0.79 \\
\hline $17 \mathrm{p} 12$ & $17: 12652500$ & MYOCD & MYOCD & rs12601765 & $\mathrm{T}$ & 0.30 & $1.04(1.03-1.06)$ & $3.08 \mathrm{E}-08$ & 0.400 & 1.17 \\
\hline $19 \mathrm{p} 12$ & $19: 22032639$ & ZNF257 & ZNF257 & rs8105767 & G & 0.30 & $1.05(1.03-1.06)$ & 2.63E-09 & 0.143 & 1.15 \\
\hline $20 \mathrm{q} 13.31$ & 20:57441016 & CTCFL & RBM 38, BMP7 & rs13039273 & $\mathrm{C}$ & 0.46 & $1.04(1.03-1.06)$ & $3.08 \mathrm{E}-09$ & 0.874 & 1.22 \\
\hline $20 \mathrm{q} 13.33$ & 20:63638397 & STMN3 & $S L C 2 A 4 R G$ & rs75691080 & $\mathrm{T}$ & 0.10 & $0.92(0.90-0.95)$ & 7.79E-11 & 0.044 & 1.25 \\
\hline $21 \mathrm{q} 22.12$ & $21: 35072824$ & RUNXI & RUNX1 & rs2834747 & G & 0.30 & $0.96(0.94-0.97)$ & $1.44 \mathrm{E}-08$ & 0.711 & 1.17 \\
\hline $22 \mathrm{q} 12.3$ & $22: 36287509$ & MYH9, APOL1 & МYH9 & rs9610482 & $\mathrm{T}$ & 0.19 & $1.06(1.04-1.08)$ & $7.89 \mathrm{E}-11$ & 0.354 & 0.94 \\
\hline \multicolumn{11}{|c|}{ Novel loci in META-2 } \\
\hline $2 \mathrm{p} 11.2$ & $2: 86458018$ & KDM3A & KDM3A & rs573520030 & $\mathrm{T}$ & 0.99 & $0.62(0.53-0.74)$ & $3,87 \mathrm{E}-08$ & 0.918 & 1.83 \\
\hline $4 \mathrm{p} 11$ & 4:49085558 & $\mathrm{CWH} 43$ & OCIADI & rs538533131 & A & 0.99 & $0.53(0.43-0.65)$ & 2,61E-09 & 0.002 & 1.15 \\
\hline $10 \mathrm{q} 25.1$ & $10: 106821670$ & SORCS1 & SORCS1 & rs 12247648 & $\mathrm{~T}$ & 0.03 & $1.26(1.17-1.36)$ & $3,86 \mathrm{E}-09$ & 0.825 & 39.55 \\
\hline Xp11.23 & $X: 46904059$ & $J A D E 3, R P 2$ & SLC9A7 & rs6611312 & $\mathrm{T}$ & 0.53 & $1.05(1.03-1.07)$ & $2,54 \mathrm{E}-08$ & 0.355 & 1.07 \\
\hline
\end{tabular}

5 Chr, chromosome; Pos, position (build 38); EA, effect allele; EAF, effect allele frequency; OR, odds ratio; CI, confidence interval; P, p-value; HetPVal, p-value for heterogeneity; FIN enr,

6 Finnish enrichment (calculated as FIN AF / NFEE AF in the Genome Aggregation Database (gnomAD), where FIN AF is the Finnish allele frequency and NFEE AF is the non-Finnish-non8 window around the association lead variant. 
medRxiv preprint doi: https://doi.org/10.1101/2021.08.20.21262098; this version posted August 25, 2021. The copyright holder for this preprint (which was not certified by peer review) is the author/funder, who has granted medRxiv a license to display the preprint in perpetuity.

It is made available under a CC-BY-ND 4.0 International license.

190 Figure 1. A combined Manhattan plot of UL associations in META-1 and META-2.

191 We conducted UL GWAS in FinnGen and, subsequently, two sets of meta-analyses with data 192 from a previously published UL meta-analysis ${ }^{2}$ and the UK Biobank, adding up to 53,534 UL 193 cases and 373,024 female controls in META-1 (top) and 33,244 UL cases and 311,271 194 female controls in META-2 (bottom), respectively. Blue color denotes novel UL risk loci 195 identified in META-1, and red color indicates novel loci identified in META-2 that were not 196 associated previously with UL risk in prior GWASs or META-1. Black and grey colours 197 indicate odd and even chromosome numbers, respectively. The red dashed lines correspond 198 to the threshold for genome-wide significance $\left(p<5 \times 10^{-8}\right)$.

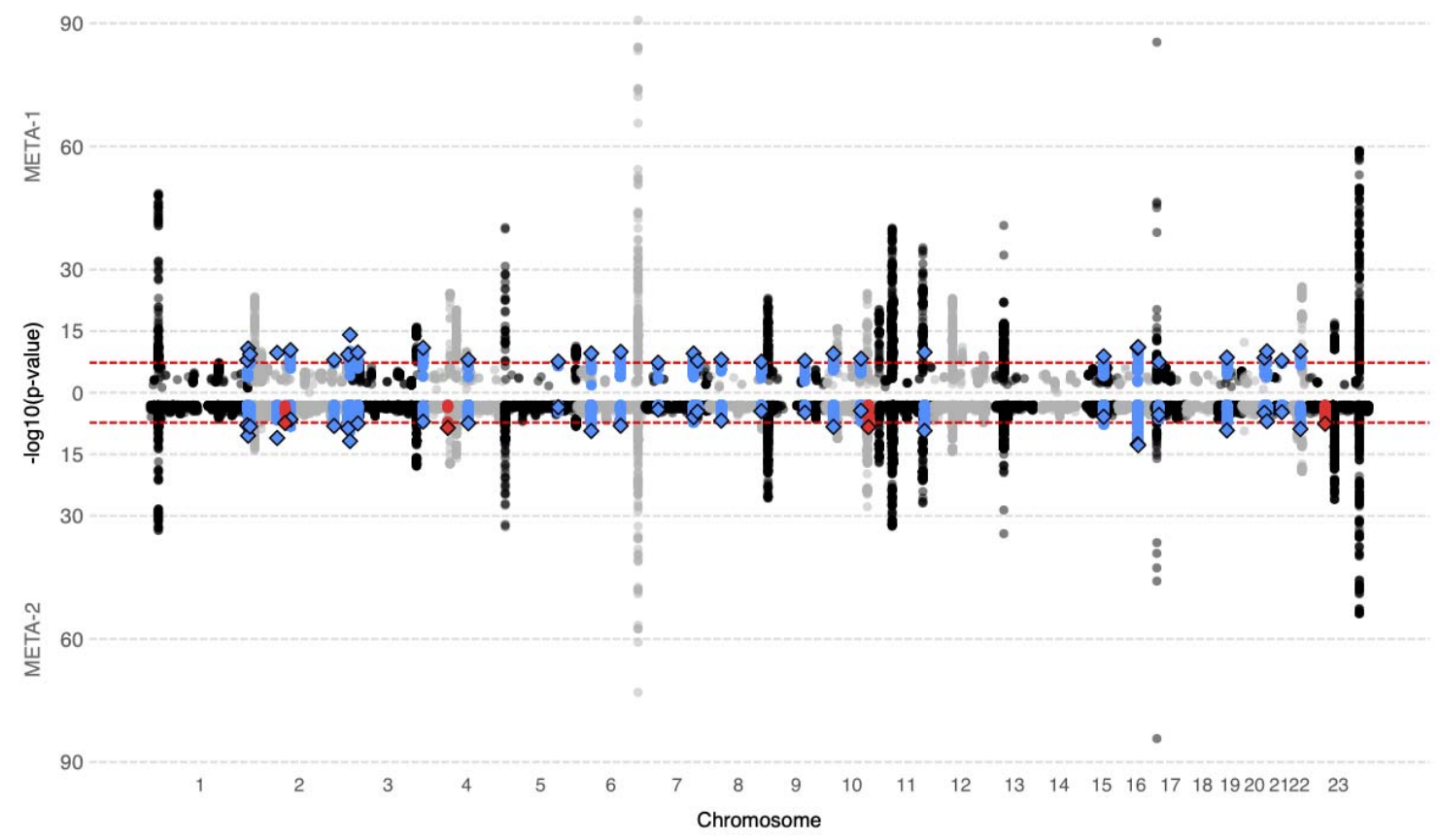


medRxiv preprint doi: https://doi.org/10.1101/2021.08.20.21262098; this version posted August 25, 2021. The copyright holder for this preprint (which was not certified by peer review) is the author/funder, who has granted medRxiv a license to display the preprint in perpetuity.

It is made available under a CC-BY-ND 4.0 International license .

199 Figure 2. Variant summary and gene set-based results using summary statistics from 200 META-2.

201 A) The proportions of 'independent genome-wide significant variants' and 'variants in LD 202 with independent significant variants' having corresponding functional annotation. Bars are 203 colored according to $-\log _{2}$ (enrichment) relative to all variants in the reference panel. B) A 204 Manhattan plot of the gene-based test computed by MAGMA ${ }^{54}$. The input variants were 205 mapped to 19,920 protein coding genes and, thus, significance was considered at $p<2.51 \times 10^{-6}$ $206(0.05 / 19,920)$; only 87 genes with p-values below this threshold are plotted. Blue and red 207 colours indicate odd and even chromosome numbers, respectively. C) MAGMA ${ }^{54}$ gene-set 208 enrichment analysis was performed for curated gene sets and GO terms available at $209 \mathrm{MsigDB}^{55}$. The plot shows the results for significantly enriched pathways $\left(p_{\text {Bonferroni }}<0.05\right)$. 210 D) Results of MAGMA ${ }^{54}$ tissue expression analysis testing a positive relationship between 211 tissue-specific gene expression profiles and disease-gene associations. Tissue-specific gene212 expression data were from GTEx (v8 $)^{56}$. All data plotted in Figure 2 A-D were produced 213
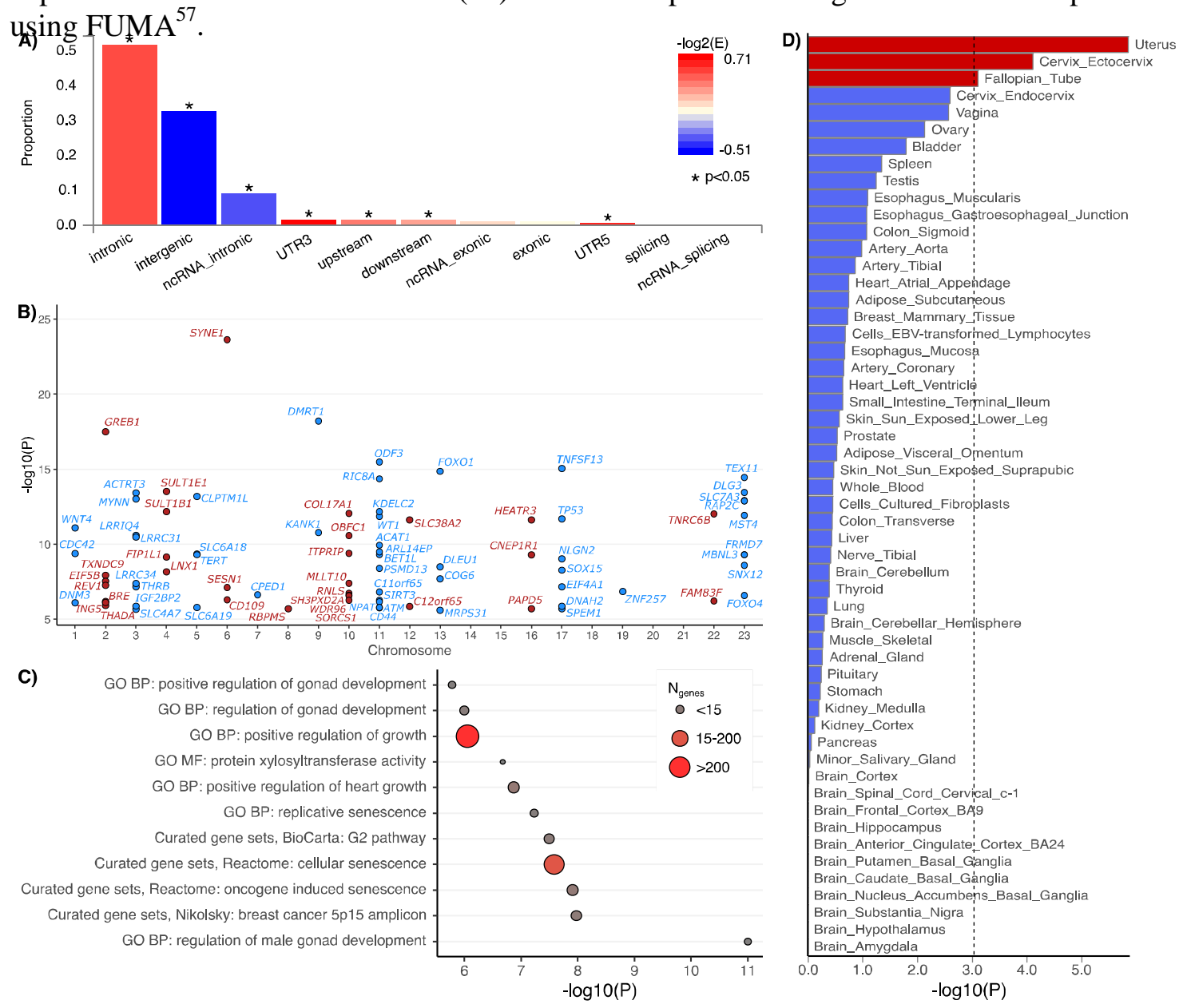
medRxiv preprint doi: https://doi.org/10.1101/2021.08.20.21262098; this version posted August 25, 2021. The copyright holder for this preprint (which was not certified by peer review) is the author/funder, who has granted medRxiv a license to display the preprint in perpetuity.

It is made available under a CC-BY-ND 4.0 International license .

\section{Figure 3. Genetic correlations ( $\left.\mathbf{r}_{\mathrm{g}}\right)$ of UL with other traits.}

215 The analyses were completed using an automated LD score regression pipeline available at 216 http://ldsc.broadinstitute.org $/^{43}$. Statistical significance was considered at $p<6 \times 10^{-5}(0.05 / 830$, 217 where 830 is the number of traits tested). The results of traits that showed significant $r_{g}$ with 218 UL are plotted (more results are provided in Table S8). Traits are named as reported in the 219 LD Hub output.

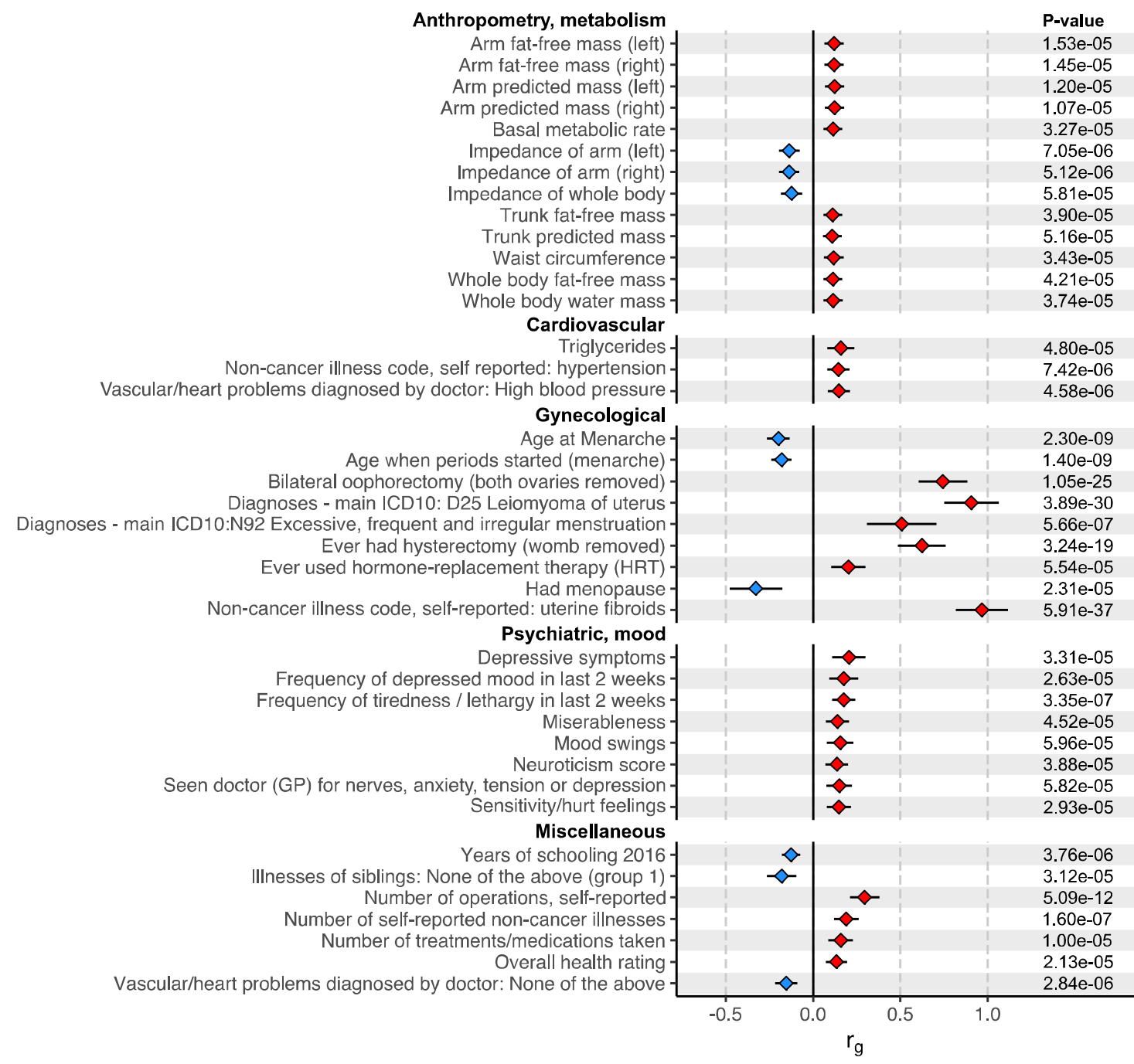


medRxiv preprint doi: https://doi.org/10.1101/2021.08.20.21262098; this version posted August 25, 2021. The copyright holder for this preprint (which was not certified by peer review) is the author/funder, who has granted medRxiv a license to display the preprint in perpetuity.

It is made available under a CC-BY-ND 4.0 International license.

\section{Figure 4. Causal relationships between UL and body composition measures.}

221 We estimated causal relationships between UL and the key traits showing significant genetic 222 correlation with UL using two-sample Mendelian randomization implemented in 223 'TwoSampleMR' R library ${ }^{58,59}$. Bi-directional causal estimates were obtained by using summary statistics provided by the MRC Integrative Epidemiology Unit (IEU) GWAS database and UL GWAS results obtained in FinnGen in the present study to extract genetic instruments for other, mostly UKBB-based, traits and UL, respectively. LD pruning was completed using European population reference, threshold of $\mathrm{r}^{2}=0.001$, and clumping window of $10 \mathrm{~kb}$. Statistical significance was considered at $p<3 \times 10^{-3}(0.05 / 19$, where 19 is the number of traits tested; black diamonds). The plot shows the causal estimates obtained using inverse variance weighted method for key body composition measures (more results are provided in Table S9; the scatter plots for the body composition measures can be found in Figures S43-S49, and the results of the leave-one-out sensitivity analyses are showin in Figures S50-S A) Exposure to LM
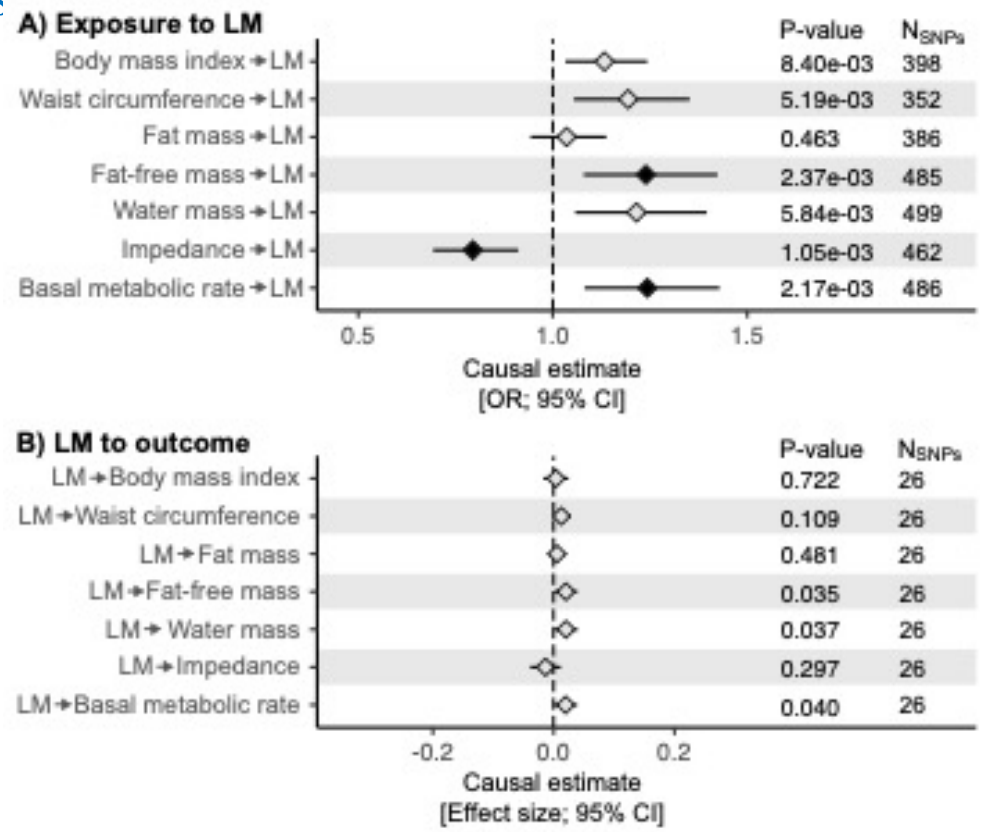
medRxiv preprint doi: https://doi.org/10.1101/2021.08.20.21262098; this version posted August 25, 2021. The copyright holder for this preprint (which was not certified by peer review) is the author/funder, who has granted medRxiv a license to display the preprint in perpetuity.

It is made available under a CC-BY-ND 4.0 International license .

\section{Methods}

\section{Study populations}

236 FinnGen (www.finngen.fi/en) is a public-private partnership project launched in 2017 with 237 an aim to improve human health through genetic research. The project utilizes genome 238 information from a nationwide network of Finnish biobanks that are linked with digital health 239 records from national hospital discharge (available from 1968), death (1969-), cancer (1953240 ), and medication reimbursement (1995-) registries using the unique national personal 241 identification codes. Ultimately, the data resource will cover roughly $10 \%$ of the Finnish 242 population. We studied data from 123,579 female participants (18,060 UL cases and 105,519 243 female controls) from FinnGen Preparatory Phase Data Freeze 5. UL cases were required to 244 have an entry of ICD-10: D25, ICD-9: 218, or ICD-8: 21899, and participants who had no records of these entries were deemed as controls. The Coordinating Ethics Committee of the Hospital District of Helsinki and Uusimaa (HUS) approved the FinnGen study protocol $\mathrm{Nr}$ HUS/990/2017.

The FinnGen study is approved by Finnish Institute for Health and Welfare (THL), approval number THL/2031/6.02.00/2017, amendments THL/1101/5.05.00/2017, THL/341/6.02.00/2018, THL/2222/6.02.00/2018, THL/283/6.02.00/2019, 251 THL/1721/5.05.00/2019, Digital and population data service agency VRK43431/2017-3, 252 VRK/6909/2018-3, VRK/4415/2019-3 the Social Insurance Institution (KELA) KELA 253 58/522/2017, KELA 131/522/2018, KELA 70/522/2019, KELA 98/522/2019, and Statistics 254 Finland TK-53-1041-17.

The Biobank Access Decisions for FinnGen samples and data utilized in FinnGen Data Freeze 5 include: THL Biobank BB2017_55, BB2017_111, BB2018_19, BB_2018_34, BB_2018_67, BB2018_71, BB2019_7, BB2019_8, BB2019_26, Finnish Red Cross Blood Service Biobank 7.12.2017, Helsinki Biobank HUS/359/2017, Auria Biobank AB17-5154, Biobank Borealis of Northern Finland_2017_1013, Biobank of Eastern Finland 1186/2018, Finnish Clinical Biobank Tampere MH0004, Central Finland Biobank 1-2017, and Terveystalo Biobank STB 2018001.

FibroGENE is consortium of conventional, population-based and direct-to-consumer cohorts that was assembled to replicate and identify UL risk variants. In the study by Gallagher et al., they studied data from 35,474 UL cases and 267,505 female controls including participants from four population-based cohorts (Women's Genome Health Study, WGHS; Northern Finland Birth Cohort, NFBC; QIMR Berghofer Medical Research Institute, QIMR; the UK Biobank, UKBB) and one direct-to-consumer cohort (23andMe) Detailed descriptions of cohorts and sample selections are available in Supplementary Methods ${ }^{2}$.

269 The UK Biobank is a large national and international health resource following the health and wellbeing of 500,000 male and female volunteer participants, enrolled at ages from 40 to $69^{60}$. The study began in 2006 with the aim to follow the participants for at least 30 years thereafter. Information has been collected from participants during recruitment using questionnaires on socioeconomic status, lifestyle, family history and medical history. Participants have also been followed up for cause-specific morbidity and mortality through linkage to disease registries, death registries, and hospital admission records. For this study, altogether 220,936 women of European ancestry were considered. Based on both hospitallinked medical records and self-report (interview with research nurse), women with a history of UL were selected as cases $(n=15,184)$, while controls $(n=205,752)$ had no previous history of UL. Informed consent was obtained from all participants. The UKBB project is approved by the North West Multi-Centre Research Ethics Committee. 
282 In FinnGen, genotyping of the samples was performed using Illumina and Affymetrix arrays 283 (Illumina Inc., San Diego, and Thermo Fisher Scientific, Santa Clara, CA, USA). Sample 284 quality control (QC) was performed to exclude individuals with high genotype missingness $(>5 \%)$, ambiguous gender, excess heterozygosity $( \pm 4 \mathrm{SD})$ and non-Finnish ancestry. Regarding variant QC, all variants with low Hardy-Weinberg equilibrium (HWE) p-value $(<1 \mathrm{e}-6)$, high missingness $(>2 \%)$ and minor allele count $($ MAC) $<3$ were excluded. Chip genotyped samples were pre-phased with Eagle 2.3.5 with the number of conditioning haplotypes set to 20,000. Genotype imputation was carried out by using the Finnish population-specific SISu v3 reference panel with Beagle 4.1 (version 08Jun17.d8b) as described in the following protocol: dx.doi.org/10.17504/protocols.io.nmndc5e. In postimputation QC, variants with imputation INFO $<0.6$ were excluded.

293 In UK Biobank, genotyping of samples was performed either on the Affymetrix UK BiLEVE 294 or Affymetrix UK Biobank Axiom array. The UK Biobank genotype data passed centralised quality control and was imputed by the UKBB team up to the HRC reference panel ${ }^{61}$.

297 The UL GWAS in FinnGen was completed using the Scalable and Accurate Implementation 298 of Generalized (SAIGE) software ${ }^{62}$. The association models were adjusted for age, sex, the 299 first 10 genetic principal components, and genotyping batch, and only variants with minimum 300 allele count of 5 were included in the analysis.

301 Given the extensive relatedness in the UK Biobank data, a linear mixed model (LMM) was 302 utilised for association testing that accounts for population structure and model the related 303 individuals, including genotyping array, age and BMI as covariates as implemented in Bolt-

\section{LMM V2.3.}

\section{Meta-analyses}

306 Two sets of fixed-effect, inverse-variance weighted meta-analyses (implemented in 307 METAL ${ }^{63}$ ) were performed: the results obtained in FinnGen were meta-analyzed with 1) the 308 top 10,000 most significant variants associating with UL in a published GWAS ${ }^{2}$ (META-1) 309 and 2) UL GWAS results obtained in the UKBB. Statistical significance was considered at 310 the standard genome-wide significance level $\left(p<5 \times 10^{-8}\right)$. Genomic inflation factor was 311 estimated using an automated LD score (LDSC) regression pipeline ${ }^{43}$.

\section{Characterization of association signals}

313 We used a web-based platform FUMA $^{57}$ to perform functional annotations of the GWAS 314 results: we completed functional gene mapping and gene-based association and enrichment 315 tests using the genome-wide UL associations from META-2 (FinnGen and UKBB) and 316 predefined lead variants as reported in Table S2. FUMA identifies variants showing genome317 wide significant association $\left(p<5 \times 10^{-8}\right)$ with the study trait and, among the significant 318 variants, identifies variants in low LD $\left(\mathrm{r}^{2}<0.6\right)$ as 'independent significant variants' and 319 further identifies variants in $\operatorname{LD}\left(r^{2}>0.6\right)$ with the 'independent significant variants'; ANNOVAR ${ }^{64}$ annotations are performed for all these variants to obtain information on the functional consequences of the key variants. MAGMA ${ }^{54}$, also implemented in FUMA, was used to perform gene-based association testing and gene-set enrichment analyses: gene-based p-values were computed for protein-coding genes by mapping variants to genes and subsequent enrichment analyses were performed for the significant genes using 4728 curated gene sets and $6166 \mathrm{GO}$ terms as reported in $\mathrm{MsigDB}^{55}$. 
326 To further characterize the potential UL candidate gene(s) according to biological relevance,

327 we annotated all genes within $\pm 1 \mathrm{Mb}$ window from the association lead variant. We explored 328 information provided by GenBank ${ }^{37}$ and UniProt ${ }^{65}$ to determine functions of the genes. To 329 complement the information available in these databases, a broad literature search was 330 performed to identify previous work published regarding to the genes of interest.

331 In each locus, we explored the associations of the lead variant with gene expression levels in 332 the Genotype-Tissue Expression (GTEx) Portal; GTEx was accessed during 02/10/2021$33302 / 11 / 2021$. To further test if altered gene expression mediates UL risk associations, we used 334 a method proposed by Zhu et al. ${ }^{66}$ as implemented in Complex Traits Genetics Virtual Lab 335 (CTG-VL) ${ }^{67}$; we performed these tests using genome-wide UL results from META-2 and 336 tissue-specific gene expression data (GTEx, V7) for uterus and whole blood. We further used 337 Regulome $\mathrm{DB}^{33}$ to discover regulatory elements overlapping with the intergenic variants in 338 the novel loci showing genome-wide significant association with UL risk. Here, significant 339 results for the novel loci are reported.

\section{SNP-based heritability and genetic correlations}

341 The SNP-based heritability $\left(\mathrm{h}^{2}{ }_{\text {SNP }}\right)$ of UL was estimated using LDSC regression implemented 342 in LDSC software ${ }^{68}$. Population prevalence of 0.30 (as in ${ }^{2}$ ) and sample prevalence of 0.11 343 were used to estimate $\mathrm{h}^{2}$ SNP on liability scale. We further applied LDSC to estimate genetic 344 correlation $\left(r_{g}\right)$ of UL with other traits using LD Hub ${ }^{43}$, a web interface for performing automated LDSC regression. As recommended, we excluded the major histocompatilibity complex region from the analyses ${ }^{43}$. We tested $r_{\mathrm{g}}$ between UL and all 830 traits available in the LD Hub database and, thus, statistical significance was considered at $p<6 \times 10^{-5}$ $(0.05 / 830)$.

\section{Mendelian randomization}

350 To test for causal inferences between UL and the key traits showing significant $r_{g}$ with UL, 351 we performed bi-directional two-sample Mendelian randomization. These analyses were 352 completed using 'TwoSampleMR' R library ${ }^{58}$ (https://mrcieu.github.io/TwoSampleMR/). To avoid possible bias from overlapping samples, we extracted genetic instruments for UL from the GWAS results obtained in FinnGen, and for other traits from the GWAS database provided by the MRC Integrative Epidemiology Unit (IEU) available at https://gwas.mrcieu.ac.uk/ and integrated in TwoSampleMR. LD pruning was completed using European population reference, threshold of $r^{2}=0.001$, and clumping window of $10 \mathrm{~kb}$, as set as default in 'clump_data' function; the numbers of SNPs available for the analyses are listed in Table S9. Inverse variance weighted (IVW) method was considered as a primary analysis, and MR Egger estimates were derived in sensitivity analyses: the estimates were required to be to a matching direction with the IVW-estimates and Egger intercepts were evaluated to assess horizontal pleiotropy. Cochran's Q statistics were derived using 'mr_heterogeneity' function to test for heterogeneity. To screen for highly influential variants that could drive the association for example due to horizontal pleiotropy, we performed leave-one-out analyses using 'mr_leaveoneout' function. Statistical significance for the causal effects was considered at $p<3 \times 10^{-3}(0.05 / 19)$ to correct for 19 traits tested. 
medRxiv preprint doi: https://doi.org/10.1101/2021.08.20.21262098; this version posted August 25, 2021. The copyright holder for this preprint (which was not certified by peer review) is the author/funder, who has granted medRxiv a license to display the preprint in perpetuity.

It is made available under a CC-BY-ND 4.0 International license .

\section{Acknowledgements and funding}

368 The work was supported through The Sigrid Juselius Foundation (JK) and funds from the 369 Academy of Finland [grant numbers 297338 and 307247](JK), Novo Nordisk Foundation 370 [grant number NNF17OC0026062] (JK), and The Finnish Medical Association (OU).

371 The FinnGen project is funded by two grants from Business Finland (HUS 4685/31/2016 and 372 UH 4386/31/2016) and eleven industry partners (AbbVie Inc, AstraZeneca UK Ltd, Biogen 373 MA Inc, Celgene Corporation, Celgene International II Sàrl, Genentech Inc, Merck Sharp \& 374 Dohme Corp, Pfizer Inc., GlaxoSmithKline, Sanofi, Maze Therapeutics Inc., Janssen Biotech 375 Inc). Following biobanks are acknowledged for delivering biobank samples to Finngen: 376 Auria Biobank (www.auria.fi/biopankki), THL Biobank (www.thl.fi/biobank), Helsinki 377 Biobank (www.helsinginbiopankki.fi), Biobank Borealis of Northern Finland 378 (https://www.ppshp.fi/Tutkimus-ja-opetus/Biopankki/Pages/Biobank-Borealis-briefly-in-

379 English.aspx), Finnish Clinical Biobank Tampere (www.tays.fi/en380 US/Research_and_development/Finnish_Clinical_Biobank_Tampere), Biobank of Eastern 381 Finland (www.ita-suomenbiopankki.fi/en), Central Finland Biobank (www.ksshp.fi/fi382 FI/Potilaalle/Biopankki), Finnish Red Cross Blood Service Biobank 383 (www.veripalvelu.fi/verenluovutus/biopankkitoiminta) and Terveystalo Biobank 384 (www.terveystalo.com/fi/Yritystietoa/Terveystalo-Biopankki/Biopankki/). All Finnish 385 Biobanks are members of BBMRI.fi infrastructure (www.bbmri.fi).

386 This research has been conducted using data from UK Biobank, a major biomedical database, 387 (http://www.ukbiobank.ac.uk/) under project ID 9637 (Gallagher et al., 2019).

388 The Genotype-Tissue Expression (GTEx) Project was supported by the Common Fund of the 389 Office of the Director of the National Institutes of Health, and by NCI, NHGRI, NHLBI, 390 NIDA, NIMH, and NINDS. The data used for the analyses described in this manuscript were 391 obtained from the GTEx Portal on 02/10/2021-02/11/2021.

\section{Author contributions}

393 OU conceptualized the study. ES, NR, KTZ, CMB, and JK contributed to the analysis plan. 394 ES, JT and NR analyzed data and generated results. ES, JT, NR, KTZ, and OU interpreted 395 the results. ES and OU wrote the original manuscript. JK supervised the study, obtained 396 funding and provided additional study resources. All authors contributed to revising the 397 content of the manuscript and approved the final version.

\section{Competing interests}

399 KTZ: Competing financial interests: Scientific collaborations (grant funding) with Bayer AG, 400 Roche Diagnostics Inc, MDNA Life Sciences, and Evotec. Competing non-financial 401 interests: Board memberships of the World Endometriosis Society, World Endometriosis 402 Research Foundation, and research advisory committee member of Wellbeing of Women 403 UK.

404 CMB: Competing financial interests: Scientific collaborations (grant funding) with Bayer 405 AG, Roche Diagnostics Inc, MDNA Life Sciences, and Evotec. Scientific board Myovant; 406 IDDM Member ObsEva. Competing non-financial interests: Chair ESHRE Endometriosis 407 Guideline Development Group. 
408

409

410

411

412

413

414

415

416

417

$418 \quad 4$

419

420

421

422

423

424

425

426

427

428

429

430

431

432

433

434

435

436

437

438

439

440

441

442

443

444

445

446

447

448

449

$450 \quad 17$

451

452

453

\section{References}

1 Baird, D. D., Dunson, D. B., Hill, M. C., Cousins, D. \& Schectman, J. M. High cumulative incidence of uterine leiomyoma in black and white women: ultrasound evidence. Am. J. Obstet. Gynecol. 188, 100-107 (2003).

2 Gallagher, C. S. et al. Genome-wide association and epidemiological analyses reveal common genetic origins between uterine leiomyomata and endometriosis. Nat Commun 10, 4857, doi:10.1038/s41467-019-12536-4 (2019).

3 Abdul Ghaffar, N. A., Ismail, M. P., Nik Mahmood, N. M., Daud, K. \& Abu Dzarr, G. A. Huge uterine fibroid in a postmenopausal woman associated with polycythaemia: a case report. Maturitas 60, 177-179, doi:10.1016/j.maturitas.2008.03.013 (2008).

4 Stewart, E. A. et al. Uterine fibroids. Nat Rev Dis Primers 2, 16043, doi:10.1038/nrdp.2016.43 (2016).

5 Stewart, E. A., Cookson, C. L., Gandolfo, R. A. \& Schulze-Rath, R. Epidemiology of uterine fibroids: a systematic review. BJOG 124, 1501-1512, doi:10.1111/1471-0528.14640 (2017).

6 Pavone, D., Clemenza, S., Sorbi, F., Fambrini, M. \& Petraglia, F. Epidemiology and Risk Factors of Uterine Fibroids. Best Pract. Res. Clin. Obstet. Gynaecol. 46, 3-11, doi:10.1016/j.bpobgyn.2017.09.004 (2018).

7 Van Voorhis, B. J., Romitti, P. A. \& Jones, M. P. Family history as a risk factor for development of uterine leiomyomas. Results of a pilot study. J. Reprod. Med. 47, 663-669 (2002).

8 Luoto, R. et al. Heritability and risk factors of uterine fibroids--the Finnish Twin Cohort study. Maturitas 37, 15-26 (2000).

9 Treloar, S. A., Martin, N. G., Dennerstein, L., Raphael, B. \& Heath, A. C. Pathways to hysterectomy: insights from longitudinal twin research. Am. J. Obstet. Gynecol. 167, 82-88, doi:10.1016/s0002-9378(11)91632-9 (1992).

10 Wise, L. A. et al. African ancestry and genetic risk for uterine leiomyomata. Am. J. Epidemiol. 176, 1159-1168, doi:10.1093/aje/kws276 (2012).

11 Mehine, M., Makinen, N., Heinonen, H. R., Aaltonen, L. A. \& Vahteristo, P. Genomics of uterine leiomyomas: insights from high-throughput sequencing. Fertil. Steril. 102, 621-629, doi:10.1016/j.fertnstert.2014.06.050 (2014).

12 Cha, P. C. et al. A genome-wide association study identifies three loci associated with susceptibility to uterine fibroids. Nat. Genet. 43, 447-450, doi:10.1038/ng.805 (2011).

13 Eggert, S. L. et al. Genome-wide linkage and association analyses implicate FASN in predisposition to Uterine Leiomyomata. Am. J. Hum. Genet. 91, 621-628, doi:10.1016/j.ajhg.2012.08.009 (2012).

14 Hellwege, J. N. et al. A multi-stage genome-wide association study of uterine fibroids in African Americans. Hum. Genet. 136, 1363-1373, doi:10.1007/s00439-017-1836-1 (2017).

15 Valimaki, N. et al. Genetic predisposition to uterine leiomyoma is determined by loci for genitourinary development and genome stability. Elife 7, doi:10.7554/eLife.37110 (2018).

16 Rafnar, T. et al. Variants associating with uterine leiomyoma highlight genetic background shared by various cancers and hormone-related traits. Nat Commun 9, 3636, doi:10.1038/s41467-018-05428-6 (2018).

17 Sakai, K. et al. Identification of a novel uterine leiomyoma GWAS locus in a Japanese population. Sci. Rep. 10, 1197, doi:10.1038/s41598-020-58066-8 (2020).

18 Sepulveda, P. V., Bush, E. D. \& Baar, K. Pharmacology of manipulating lean body mass. Clin. Exp. Pharmacol. Physiol. 42, 1-13, doi:10.1111/1440-1681.12320 (2015). 
19 Ono, M. et al. Paracrine activation of WNT/beta-catenin pathway in uterine leiomyoma stem cells promotes tumor growth. Proc. Natl. Acad. Sci. U. S. A. 110, 17053-17058, doi:10.1073/pnas.1313650110 (2013).

20 Willert, K. \& Nusse, R. Wnt proteins. Cold Spring Harb. Perspect. Biol. 4, a007864, doi:10.1101/cshperspect.a007864 (2012).

21 Borahay, M. A. et al. Estrogen Receptors and Signaling in Fibroids: Role in Pathobiology and Therapeutic Implications. Reprod. Sci. 24, 1235-1244, doi:10.1177/1933719116678686 (2017).

22 Massaad-Massade, L. et al. HMGA1 enhances the transcriptional activity and binding of the estrogen receptor to its responsive element. Biochemistry 41, 2760-2768, doi:10.1021/bi011455j (2002).

23 Dhamad, A. E., Zhou, Z., Zhou, J. \& Du, Y. Systematic Proteomic Identification of the Heat Shock Proteins (Hsp) that Interact with Estrogen Receptor Alpha (ERalpha) and Biochemical Characterization of the ERalpha-Hsp70 Interaction. PLoS One 11, e0160312, doi:10.1371/journal.pone.0160312 (2016).

24 Prossnitz, E. R. et al. Estrogen signaling through the transmembrane G protein-coupled receptor GPR30. Аnпи. Rev. Physiol. 70, 165-190, doi:10.1146/annurev.physiol.70.113006.100518 (2008).

25 Ciarmela, P. et al. Growth factors and myometrium: biological effects in uterine fibroid and possible clinical implications. Hum. Reprod. Update 17, 772-790, doi:10.1093/humupd/dmr031 (2011).

26 Peng, L. et al. Expression of insulin-like growth factors (IGFs) and IGF signaling: molecular complexity in uterine leiomyomas. Fertil. Steril. 91, 2664-2675, doi:10.1016/j.fertnstert.2007.10.083 (2009).

27 Perrot, V. \& Rechler, M. M. The coactivator p300 directly acetylates the forkhead transcription factor Foxo1 and stimulates Foxo1-induced transcription. Mol. Endocrinol. 19, 2283-2298, doi:10.1210/me.2004-0292 (2005).

28 Guo, S. et al. Phosphorylation of serine 256 by protein kinase B disrupts transactivation by FKHR and mediates effects of insulin on insulin-like growth factor-binding protein-1 promoter activity through a conserved insulin response sequence. J. Biol. Chem. 274, 1718417192, doi:10.1074/jbc.274.24.17184 (1999).

29 Lewis, T. D. et al. Ulipristal acetate decreases active TGF-beta3 and its canonical signaling in uterine leiomyoma via two novel mechanisms. Fertil. Steril. 111, 806-815 e801, doi:10.1016/j.fertnstert.2018.12.026 (2019).

30 Chen, J., Kitchen, C. M., Streb, J. W. \& Miano, J. M. Myocardin: a component of a molecular switch for smooth muscle differentiation. J. Mol. Cell. Cardiol. 34, 1345-1356, doi:10.1006/jmcc.2002.2086 (2002).

31 Liao, X. H. et al. ERalpha inhibited myocardin-induced differentiation in uterine fibroids. Exp. Cell Res. 350, 73-82, doi:10.1016/j.yexcr.2016.11.007 (2017).

32 Zhao, J. et al. MYOSLID Is a Novel Serum Response Factor-Dependent Long Noncoding RNA That Amplifies the Vascular Smooth Muscle Differentiation Program. Arterioscler. Thromb. Vasc. Biol. 36, 2088-2099, doi:10.1161/ATVBAHA.116.307879 (2016).

33 Boyle, A. P. et al. Annotation of functional variation in personal genomes using RegulomeDB. Genome Res. 22, 1790-1797, doi:10.1101/gr.137323.112 (2012).

34 Raimundo, N., Vanharanta, S., Aaltonen, L. A., Hovatta, I. \& Suomalainen, A. Downregulation of SRF-FOS-JUNB pathway in fumarate hydratase deficiency and in uterine leiomyomas. Oncogene 28, 1261-1273, doi:10.1038/onc.2008.472 (2009). 
Edwards, T. L., Hartmann, K. E. \& Velez Edwards, D. R. Variants in BET1L and TNRC6B associate with increasing fibroid volume and fibroid type among European Americans. Hum. Genet. 132, 1361-1369, doi:10.1007/s00439-013-1340-1 (2013).

36 Masuda, T. et al. GWAS of five gynecologic diseases and cross-trait analysis in Japanese. Eur. J. Hum. Genet. 28, 95-107, doi:10.1038/s41431-019-0495-1 (2020).

37 Clark, K., Karsch-Mizrachi, I., Lipman, D. J., Ostell, J. \& Sayers, E. W. GenBank. Nucleic Acids Res. 44, D67-72, doi:10.1093/nar/gkv1276 (2016).

38 Mehine, M. et al. Integrated data analysis reveals uterine leiomyoma subtypes with distinct driver pathways and biomarkers. Proc. Natl. Acad. Sci. U. S. A. 113, 1315-1320, doi:10.1073/pnas.1518752113 (2016).

39 Kimura, Y., Morita, T., Hayashi, K., Miki, T. \& Sobue, K. Myocardin functions as an effective inducer of growth arrest and differentiation in human uterine leiomyosarcoma cells. Cancer Res. 70, 501-511, doi:10.1158/0008-5472.CAN-09-1469 (2010).

40 Markowski, D. N. et al. HMGA2 and the p19Arf-TP53-CDKN1A axis: a delicate balance in the growth of uterine leiomyomas. Genes Chromosomes Cancer 49, 661-668, doi:10.1002/gcc.20777 (2010).

41 Staley, J. R. et al. PhenoScanner: a database of human genotype-phenotype associations. Bioinformatics 32, 3207-3209, doi:10.1093/bioinformatics/btw373 (2016).

42 Kamat, M. A. et al. PhenoScanner V2: an expanded tool for searching human genotypephenotype associations. Bioinformatics 35, 4851-4853, doi:10.1093/bioinformatics/btz469 (2019).

43 Zheng, J. et al. LD Hub: a centralized database and web interface to perform LD score regression that maximizes the potential of summary level GWAS data for SNP heritability and genetic correlation analysis. Bioinformatics 33, 272-279, doi:10.1093/bioinformatics/btw613 (2017).

44 Marshall, L. M. et al. Risk of uterine leiomyomata among premenopausal women in relation to body size and cigarette smoking. Epidemiology 9, 511-517 (1998).

45 Wise, L. A. et al. Influence of body size and body fat distribution on risk of uterine leiomyomata in U.S. black women. Epidemiology 16, 346-354 (2005).

46 Lee, J. E. et al. Weight change and risk of uterine leiomyomas: Korea Nurses' Health Study. Curr. Med. Res. Opin. 34, 1913-1919, doi:10.1080/03007995.2018.1462783 (2018).

47 Bakas, P. et al. Estrogen receptor alpha and beta in uterine fibroids: a basis for altered estrogen responsiveness. Fertil. Steril. 90, 1878-1885, doi:10.1016/j.fertnstert.2007.09.019 (2008).

48 Valladares, F. et al. Characterization of estrogen receptors alpha and beta in uterine leiomyoma cells. Fertil. Steril. 86, 1736-1743, doi:10.1016/j.fertnstert.2006.05.047 (2006).

49 Sipila, S. \& Poutamo, J. Muscle performance, sex hormones and training in peri-menopausal and post-menopausal women. Scand. J. Med. Sci. Sports 13, 19-25, doi:10.1034/j.16000838.2003.20210.x (2003).

Wood, C. L., Lane, L. C. \& Cheetham, T. Puberty: Normal physiology (brief overview). Best Pract. Res. Clin. Endocrinol. Metab. 33, 101265, doi:10.1016/j.beem.2019.03.001 (2019).

1 Barros, R. P. \& Gustafsson, J. A. Estrogen receptors and the metabolic network. Cell Metab 14, 289-299, doi:10.1016/j.cmet.2011.08.005 (2011).

52 Chidi-Ogbolu, N. \& Baar, K. Effect of Estrogen on Musculoskeletal Performance and Injury Risk. Front. Physiol. 9, 1834, doi:10.3389/fphys.2018.01834 (2018). 
medRxiv preprint doi: https://doi.org/10.1101/2021.08.20.21262098; this version posted August 25, 2021. The copyright holder for this preprint (which was not certified by peer review) is the author/funder, who has granted medRxiv a license to display the preprint in perpetuity.

It is made available under a CC-BY-ND 4.0 International license .

$54653 \quad$ Velders, M. \& Diel, P. How sex hormones promote skeletal muscle regeneration. Sports Med. 43, 1089-1100, doi:10.1007/s40279-013-0081-6 (2013).

de Leeuw, C. A., Mooij, J. M., Heskes, T. \& Posthuma, D. MAGMA: generalized gene-set analysis of GWAS data. PLoS Comput. Biol. 11, e1004219, doi:10.1371/journal.pcbi.1004219 (2015).

Liberzon, A. et al. Molecular signatures database (MSigDB) 3.0. Bioinformatics 27, 17391740, doi:10.1093/bioinformatics/btr260 (2011).

Consortium, G. T. The Genotype-Tissue Expression (GTEx) project. Nat. Genet. 45, 580-585, doi:10.1038/ng.2653 (2013).

556 annotation of genetic associations with FUMA. Nat Commun 8, 1826, doi:10.1038/s41467017-01261-5 (2017).

Hemani, G. et al. The MR-Base platform supports systematic causal inference across the human phenome. Elife 7, doi:10.7554/eLife.34408 (2018).

Hemani, G., Tilling, K. \& Davey Smith, G. Orienting the causal relationship between imprecisely measured traits using GWAS summary data. PLoS genetics 13, e1007081, doi:10.1371/journal.pgen.1007081 (2017).

Sudlow, C. et al. UK biobank: an open access resource for identifying the causes of a wide range of complex diseases of middle and old age. PLoS Med. 12, e1001779, doi:10.1371/journal.pmed.1001779 (2015).

Bycroft, C. et al. The UK Biobank resource with deep phenotyping and genomic data. Nature 562, 203-209, doi:10.1038/s41586-018-0579-z (2018).

Zhou, W. et al. Efficiently controlling for case-control imbalance and sample relatedness in large-scale genetic association studies. Nat. Genet. 50, 1335-1341, doi:10.1038/s41588-0180184-y (2018).

63 Willer, C. J., Li, Y. \& Abecasis, G. R. METAL: fast and efficient meta-analysis of genomewide association scans. Bioinformatics 26, 2190-2191, doi:10.1093/bioinformatics/btq340 (2010).

64 Wang, K., Li, M. \& Hakonarson, H. ANNOVAR: functional annotation of genetic variants from high-throughput sequencing data. Nucleic Acids Res. 38, e164, doi:10.1093/nar/gkq603 (2010).

UniProt, C. UniProt: a worldwide hub of protein knowledge. Nucleic Acids Res. 47, D506D515, doi:10.1093/nar/gky1049 (2019).

Zhu, Z. et al. Integration of summary data from GWAS and eQTL studies predicts complex trait gene targets. Nat. Genet. 48, 481-487, doi:10.1038/ng.3538 (2016). platform for post-GWAS analyses. bioRxiv, 518027, doi:10.1101/518027 (2019). 


\section{Contributors of FinnGen}

588 Steering Committee

Aarno Palotie

Mark Daly

Bridget Riley-Gills

Howard Jacob

Dirk Paul

Heiko Runz

Sally John

Robert Plenge

Joseph Maranville

Mark McCarthy

Julie Hunkapiller

Meg Ehm

Kirsi Auro

Simonne Longerich

Caroline Fox

Anders Mälarstig

Katherine Klinger

Eric Green

Robert Graham

Robert Yang

Chris O’Donnell

Tomi Mäkelä

Jaakko Kaprio

Petri Virolainen

Antti Hakanen

Terhi Kilpi

Markus Perola

Jukka Partanen

Helsinki, Finland

Anne Pitkäranta

Helsinki

Juhani Junttila

Raisa Serpi

Tarja Laitinen

Tampere, Finland

Veli-Matti Kosma

Kuopio, Finland

Urho Kujala

Jyväskylä, Finland

Marco Hautalahti

Outi Tuovila

Raimo Pakkanen

Jeffrey Waring

Bridget Riley-Gillis

Ioanna Tachmazidou

Chia-Yen Chen

Heiko Runz

Shameek Biswas

Sarah Pendergrass

Julie Hunkapiller

Meg Ehm

David Pulford

Neha Raghavan

\section{Pharmaceutical companies}

Deepak Raipal

\section{University of Helsinki \& Biobanks}

District, Oulu, Finland

District, Oulu, Finland

\section{Other Experts/ Non-Voting Members}

Business Finland, Helsinki, Finland

Scientific Committee

Pharmaceutical companies

Abbvie, Chicago, IL, United States

Sanofi, Paris, France

Janssen Biotech, Beerse, Belgium

Novartis, Basel, Switzerland
Institute for Molecular Medicine Finland, HiLIFE, University of Helsinki, Finland

Astra Zeneca, Cambridge, United Kingdom

Biogen, Cambridge, MA, United States

Biogen, Cambridge, MA, United States

Celgene, Summit, NJ, United States/Bristol Myers Squibb, New York, NY, United States

Genentech, San Francisco, CA, United States

ech, San Francisco, CA, United States

GlaxoSmithKline, Brentford, United Kingdom

Merck, Kenilworth, NJ, United States

Merck, Kenilworth, NJ, United States

Maze Therapeutics, San Francisco, CA, United States

Maze Therapeutics, San Francisco, CA, United States

HiLIFE, University of Helsinki, Finland, Finland

Institute for Molecular Medicine Finland, HiLIFE, Helsinki, Finland, Finland

Auria Biobank / University of Turku / Hospital District of Southwest Finland, Turku, Finland

Auria Biobank / University of Turku / Hospital District of Southwest Finland, Turku, Finland

THL Biobank / The National Institute of Health and Welfare Helsinki, Finland

THL Biobank / The National Institute of Health and Welfare Helsinki, Finland

Finnish Red Cross Blood Service / Finnish Hematology Registry and Clinical Biobank,

Helsinki Biobank / Helsinki University and Hospital District of Helsinki and Uusimaa,

Northern Finland Biobank Borealis / University of Oulu / Northern Ostrobothnia Hospital

Northern Finland Biobank Borealis / University of Oulu / Northern Ostrobothnia Hospital

Finnish Clinical Biobank Tampere / University of Tampere / Pirkanmaa Hospital District,

Biobank of Eastern Finland / University of Eastern Finland / Northern Savo Hospital District,

Central Finland Biobank / University of Jyväskylä / Central Finland Health Care District,

FINBB - Finnish biobank cooperative
Abbvie, Chicago, IL, United States

Abbvie, Chicago, IL, United States

Astra Zeneca, Cambridge, United Kingdom

Biogen, Cambridge, MA, United States

Biogen, Cambridge, MA, United States

Celgene, Summit, NJ, United States/Bristol Myers Squibb, New York, NY, United States

Genentech, San Francisco, CA, United States

Genentech, San Francisco, CA, United States

GlaxoSmithKline, Brentford, United Kingdom

GlaxoSmithKline, Brentford, United Kingdom

Merck, Kenilworth, NJ, United States 
Merck, Kenilworth, NJ, United States

Anders Mälarstig

Xinli $\mathrm{Hu}$

658 Katherine Klinger

659 Matthias Gossel

660 Robert Graham

661 Eric Green

662 Sahar Mozaffari

663 Dawn Waterworth

664 Nicole Renaud

665 Ma'en Obeidat

Merck, Kenilworth, NJ, United States

Pfizer, New York, NY, United States

Pfizer, New York, NY, United States

Sanofi, Paris, France

Sanofi, Paris, France

Maze Therapeutics, San Francisco, CA, United States

Maze Therapeutics, San Francisco, CA, United States

Maze Therapeutics, San Francisco, CA, United States

Janssen Research \& Development, LLC, Spring House, PA, United States

Novartis, Basel, Switzerland

Novartis, Basel, Switzerland

667

668

University of Helsinki \& Biobanks

Johanna Schleutker

670 Markus Perola

671 Mikko Arvas

672 Helsinki, Finland

673 Olli Carpén

674 Helsinki

675 Reetta Hinttala

676 District, Oulu, Finland

677 Johannes Kettunen

678 District, Oulu, Finland

679 Arto Mannermaa

680 Kuopio, Finland

681 Katriina Aalto-Setälä

682 Tampere, Finland

683 Jari Laukkanen

684 Jyväskylä, Finland

685 Urho Kujala

686 Jyväskylä, Finland

687 Johanna Mäkelä

688

689

690

\section{Clinical Groups}

Neurology Group

691 Reetta Kälviäinen

692 Valtteri Julkunen

693 Hilkka Soininen

694 Anne Remes

695 Mikko Hiltunen

696 Jukka Peltola

697 Minna Raivio

698 Pentti Tienari

699 Juha Rinne

700 Roosa Kallionpää

701 Juulia Partanen

702 Ali Abbasi

703 Adam Ziemann

704 Jeffrey Waring

705 Sahar Esmaeeli

706 Nizar Smaoui

707 Anne Lehtonen

708 Susan Eaton

709 Heiko Runz

710 Sanni Lahdenperä

711 Janet van Adelsberg

712 Shameek Biswas

713 Julie Hunkapiller

714 Natalie Bowers

715 Edmond Teng

716 Sarah Pendergrass

717 Fanli Xu

718 David Pulford

719 Kirsi Auro

720 Laura Addis

Institute for Molecular Medicine Finland, HiLIFE, Helsinki, Finland

Auria Biobank / Univ. of Turku / Hospital District of Southwest Finland, Turku, Finland

THL Biobank / The National Institute of Health and Welfare Helsinki, Finland

Finnish Red Cross Blood Service / Finnish Hematology Registry and Clinical Biobank,

Helsinki Biobank / Helsinki University and Hospital District of Helsinki and Uusimaa,

Northern Finland Biobank Borealis / University of Oulu / Northern Ostrobothnia Hospital

Northern Finland Biobank Borealis / University of Oulu / Northern Ostrobothnia Hospital

Biobank of Eastern Finland / University of Eastern Finland / Northern Savo Hospital District,

Finnish Clinical Biobank Tampere / University of Tampere / Pirkanmaa Hospital District,

Central Finland Biobank / University of Jyväskylä / Central Finland Health Care District,

Central Finland Biobank / University of Jyväskylä / Central Finland Health Care District,

FINBB - Finnish biobank cooperative

Northern Savo Hospital District, Kuopio, Finland

Northern Savo Hospital District, Kuopio, Finland

Northern Savo Hospital District, Kuopio, Finland

Northern Ostrobothnia Hospital District, Oulu, Finland

Northern Savo Hospital District, Kuopio, Finland

Pirkanmaa Hospital District, Tampere, Finland

Hospital District of Helsinki and Uusimaa, Helsinki, Finland

Hospital District of Helsinki and Uusimaa, Helsinki, Finland

Hospital District of Southwest Finland, Turku, Finland

Hospital District of Southwest Finland, Turku, Finland

Institute for Molecular Medicine Finland, HiLIFE, University of Helsinki, Finland

Abbvie, Chicago, IL, United States

Abbvie, Chicago, IL, United States

Abbvie, Chicago, IL, United States

Abbvie, Chicago, IL, United States

Abbvie, Chicago, IL, United States

Abbvie, Chicago, IL, United States

Biogen, Cambridge, MA, United States

Biogen, Cambridge, MA, United States

Biogen, Cambridge, MA, United States

Celgene, Summit, NJ, United States/ Bristol Myers Squibb, New York, NY, United States

Celgene, Summit, NJ, United States/ Bristol Myers Squibb, New York, NY, United States

Genentech, San Francisco, CA, United States

Genentech, San Francisco, CA, United States

Genentech, San Francisco, CA, United States

Genentech, San Francisco, CA, United States

GlaxoSmithKline, Brentford, United Kingdom

GlaxoSmithKline, Brentford, United Kingdom

GlaxoSmithKline, Brentford, United Kingdom

GlaxoSmithKline, Brentford, United Kingdom 
John Eicher

Qingqin S Li

Karen $\mathrm{He}$

Ekaterina Khramtsova

Beryl Cummings

Neha Raghavan

Kari Linden

Gastroenterology Group

Martti Färkkilä

Jukka Koskela

Sampsa Pikkarainen

Airi Jussila

Katri Kaukinen

Timo Blomster

Mikko Kiviniemi

Markku Voutilainen

Mark Daly

Ali Abbasi

Graham Heap

Jeffrey Waring

Nizar Smaoui

Fedik Rahimov

Anne Lehtonen

Keith Usiskin

Tim Lu

Natalie Bowers

Danny Oh

Sarah Pendergrass

Linda McCarthy

Amy Hart

Meijian Guan

Jason Miller

Kirsi Kalpala

Melissa Miller

Xinli Hu

\section{Rheumatology Group}

Kari Eklund

Antti Palomäki

Pia Isomäki

Laura Pirilä

Oili Kaipiainen-Seppänen

Johanna Huhtakangas

Nina Mars

Ali Abbasi

Jeffrey Waring

Fedik Rahimov

Apinya Lertratanakul

Nizar Smaoui

Anne Lehtonen

David Close

Marla Hochfeld

Natalie Bowers

Sarah Pendergrass

Jorge Esparza Gordillo

Kirsi Auro

Dawn Waterworth

Fabiana Farias

Kirsi Kalpala

Nan Bing

Xinli Hu

\section{Pulmonology Group}

Tarja Laitinen

Margit Pelkonen

Paula Kauppi
GlaxoSmithKline, Brentford, United Kingdom

Janssen Research \& Development, LLC, Titusville, NJ 08560, United States

Janssen Research \& Development, LLC, Spring House, PA, United States

Janssen Research \& Development, LLC, Spring House, PA, United States

Maze Therapeutics, San Francisco, CA, United States

Merck, Kenilworth, NJ, United States

Pfizer, New York, NY, United States

Hospital District of Helsinki and Uusimaa, Helsinki, Finland

Hospital District of Helsinki and Uusimaa, Helsinki, Finland

Hospital District of Helsinki and Uusimaa, Helsinki, Finland

Pirkanmaa Hospital District, Tampere, Finland

Pirkanmaa Hospital District, Tampere, Finland

Northern Ostrobothnia Hospital District, Oulu, Finland

Northern Savo Hospital District, Kuopio, Finland

Hospital District of Southwest Finland, Turku, Finland

Institute for Molecular Medicine Finland, HiLIFE, University of Helsinki, Finland

Abbvie, Chicago, IL, United States

Abbvie, Chicago, IL, United States

Abbvie, Chicago, IL, United States

Abbvie, Chicago, IL, United States

Abbvie, Chicago, IL, United States

Abbvie, Chicago, IL, United States

Celgene, Summit, NJ, United States/ Bristol Myers Squibb, New York, NY, United States

Genentech, San Francisco, CA, United States

Genentech, San Francisco, CA, United States

Genentech, San Francisco, CA, United States

Genentech, San Francisco, CA, United States

GlaxoSmithKline, Brentford, United Kingdom

Janssen Research \& Development, LLC, Spring House, PA, United States

Janssen Research \& Development, LLC, Spring House, PA, United States

Merck, Kenilworth, NJ, United States

Pfizer, New York, NY, United States

Pfizer, New York, NY, United States

Pfizer, New York, NY, United States

Hospital District of Helsinki and Uusimaa, Helsinki, Finland

Hospital District of Southwest Finland, Turku, Finland

Pirkanmaa Hospital District, Tampere, Finland

Hospital District of Southwest Finland, Turku, Finland

Northern Savo Hospital District, Kuopio, Finland

Northern Ostrobothnia Hospital District, Oulu, Finland

Institute for Molecular Medicine Finland, HiLIFE, Helsinki, Finland

Abbvie, Chicago, IL, United States

Abbvie, Chicago, IL, United States

Abbvie, Chicago, IL, United States

Abbvie, Chicago, IL, United States

Abbvie, Chicago, IL, United States

Abbvie, Chicago, IL, United States

Astra Zeneca, Cambridge, United Kingdom

Celgene, Summit, NJ, United States/ Bristol Myers Squibb, New York, NY, United States

Genentech, San Francisco, CA, United States

Genentech, San Francisco, CA, United States

GlaxoSmithKline, Brentford, United Kingdom

GlaxoSmithKline, Brentford, United Kingdom

Janssen Research \& Development, LLC, Spring House, PA, United States

Merck, Kenilworth, NJ, United States

Pfizer, New York, NY, United States

Pfizer, New York, NY, United States

Pfizer, New York, NY, United States

Pirkanmaa Hospital District, Tampere, Finland

Northern Savo Hospital District, Kuopio, Finland

Hospital District of Helsinki and Uusimaa, Helsinki, Finland 
Hannu Kankaanranta

Terttu Harju

Riitta Lahesmaa

Nizar Smaoui

Alex Mackay

Glenda Lassi

Susan Eaton

Steven Greenberg

Hubert Chen

Sarah Pendergrass

Natalie Bowers

Joanna Betts

Soumitra Ghosh

Kirsi Auro

Rajashree Mishra
Pirkanmaa Hospital District, Tampere, Finland

Northern Ostrobothnia Hospital District, Oulu, Finland

Hospital District of Southwest Finland, Turku, Finland

Abbvie, Chicago, IL, United States

Astra Zeneca, Cambridge, United Kingdom

Astra Zeneca, Cambridge, United Kingdom

Biogen, Cambridge, MA, United States

Celgene, Summit, NJ, United States/ Bristol Myers Squibb, New York, NY, United States

Genentech, San Francisco, CA, United States

Genentech, San Francisco, CA, United States

Genentech, San Francisco, CA, United States

GlaxoSmithKline, Brentford, United Kingdom

GlaxoSmithKline, Brentford, United Kingdom

GlaxoSmithKline, Brentford, United Kingdom

GlaxoSmithKline, Brentford, United Kingdom

Cardiometabolic Diseases Group

Teemu Niiranen

The National Institute of Health and Welfare Helsinki, Finland

Felix Vaura

The National Institute of Health and Welfare Helsinki, Finland

Veikko Salomaa

The National Institute of Health and Welfare Helsinki, Finland

808 Markus Juonala

809 Kaj Metsärinne

Hospital District of Southwest Finland, Turku, Finland

810 Mika Kähönen

Hospital District of Southwest Finland, Turku, Finland

Pirkanmaa Hospital District, Tampere, Finland

812 Markku Laakso

Northern Ostrobothnia Hospital District, Oulu, Finland

Northern Savo Hospital District, Kuopio, Finland

Jussi Pihlajamäki

Northern Savo Hospital District, Kuopio, Finland

Daniel Gordin

Hospital District of Helsinki and Uusimaa, Helsinki, Finland

Juha Sinisalo

Hospital District of Helsinki and Uusimaa, Helsinki, Finland

Marja-Riitta Taskinen

Hospital District of Helsinki and Uusimaa, Helsinki, Finland

Tiinamaija Tuomi

Jari Laukkanen

Hospital District of Helsinki and Uusimaa, Helsinki, Finland

Central Finland Health Care District, Jyväskylä, Finland

Amanda Elliott

Institute, Cambridge, MA, United States

Joel Rämö

Samuli Ripatti

Mary Pat Reeve

Sanni Ruotsalainen

Benjamin Challis

Dirk Paul

Keith Usiskin

Julie Hunkapiller

Natalie Bowers

Sarah Pendergrass

Audrey Chu

Kirsi Auro

Dermot Reilly

Jaakko Parkkinen

Melissa Miller

Russell Miller

\section{Oncology Group}

Tuomo Meretoja

Heikki Joensuu

Olli Carpén

Lauri Aaltonen

Johanna Mattson

Annika Auranen

Peeter Karihtala

Saila Kauppila

Päivi Auvinen

Klaus Elenius

Johanna Schleutker

Esa Pitkänen

Nina Mars

Mark Daly

Relja Popovic

Jeffrey Waring
Institute for Molecular Medicine Finland, HiLIFE, University of Helsinki, Finland / Broad

Institute for Molecular Medicine Finland, HiLIFE, University of Helsinki, Finland Institute for Molecular Medicine Finland, HiLIFE, University of Helsinki, Finland Institute for Molecular Medicine Finland, HiLIFE, University of Helsinki, Finland Institute for Molecular Medicine Finland, HiLIFE, University of Helsinki, Finland Astra Zeneca, Cambridge, United Kingdom Astra Zeneca, Cambridge, United Kingdom Celgene, Summit, NJ, United States/ Bristol Myers Squibb, New York, NY, United States Genentech, San Francisco, CA, United States Genentech, San Francisco, CA, United States Genentech, San Francisco, CA, United States GlaxoSmithKline, Brentford, United Kingdom GlaxoSmithKline, Brentford, United Kingdom Janssen Research \& Development, LLC, Boston, MA, United States Pfizer, New York, NY, United States Pfizer, New York, NY, United States Pfizer, New York, NY, United States

Hospital District of Helsinki and Uusimaa, Helsinki, Finland Hospital District of Helsinki and Uusimaa, Helsinki, Finland Hospital District of Helsinki and Uusimaa, Helsinki, Finland Hospital District of Helsinki and Uusimaa, Helsinki, Finland Hospital District of Helsinki and Uusimaa, Helsinki, Finland Pirkanmaa Hospital District, Tampere, Finland

Northern Ostrobothnia Hospital District, Oulu, Finland

Northern Ostrobothnia Hospital District, Oulu, Finland

Northern Savo Hospital District, Kuopio, Finland

Hospital District of Southwest Finland, Turku, Finland

Hospital District of Southwest Finland, Turku, Finland

Institute for Molecular Medicine Finland, HiLIFE, University of Helsinki, Finland Institute for Molecular Medicine Finland, HiLIFE, University of Helsinki, Finland Institute for Molecular Medicine Finland, HiLIFE, University of Helsinki, Finland Abbvie, Chicago, IL, United States Abbvie, Chicago, IL, United States 
Bridget Riley-Gillis

Anne Lehtonen

Jennifer Schutzman

Julie Hunkapiller

Natalie Bowers

Sarah Pendergrass

Diptee Kulkarni

Kirsi Auro

Alessandro Porello

Andrey Loboda

Heli Lehtonen

Stefan McDonough

Marika Crohns

Sauli Vuoti

\section{Opthalmology Group}

Kai Kaarniranta

Joni A Turunen

Terhi Ollila

Sanna Seitsonen

Hannu Uusitalo

Vesa Aaltonen

Hannele Uusitalo-Järvinen

Marja Luodonpää

Nina Hautala

Juha Karjalainen

Esa Pitkänen

Mengzhen Liu

Heiko Runz

Stephanie Loomis

Erich Strauss

Natalie Bowers

Hao Chen

Sarah Pendergrass

\section{Dermatology Group}

Kaisa Tasanen

Laura Huilaja

Katariina Hannula-Jouppi

Teea Salmi

Sirkku Peltonen

Leena Koulu

Nizar Smaoui

Fedik Rahimov

Anne Lehtonen

David Choy

Sarah Pendergrass

Dawn Waterworth

Kirsi Kalpala

Ying $\mathrm{Wu}$

\section{Odontology Group}

Pirkko Pussinen

Aino Salminen

Tuula Salo

David Rice

Pekka Nieminen

Ulla Palotie

Juha Sinisalo

Maria Siponen

Liisa Suominen

Päivi Mäntylä

Ulvi Gursoy

Vuokko Anttonen

Kirsi Sipilä

Sarah Pendergrass
Abbvie, Chicago, IL, United States

Abbvie, Chicago, IL, United States

Genentech, San Francisco, CA, United States

Genentech, San Francisco, CA, United States

Genentech, San Francisco, CA, United States

Genentech, San Francisco, CA, United States

GlaxoSmithKline, Brentford, United Kingdom

GlaxoSmithKline, Brentford, United Kingdom

Janssen Research \& Development, LLC, Spring House, PA, United States

Merck, Kenilworth, NJ, United States

Pfizer, New York, NY, United States

Pfizer, New York, NY, United States

Sanofi, Paris, France

Sanofi, Paris, France

Northern Savo Hospital District, Kuopio, Finland

Hospital District of Helsinki and Uusimaa, Helsinki, Finland

Hospital District of Helsinki and Uusimaa, Helsinki, Finland

Hospital District of Helsinki and Uusimaa, Helsinki, Finland

Pirkanmaa Hospital District, Tampere, Finland

Hospital District of Southwest Finland, Turku, Finland

Pirkanmaa Hospital District, Tampere, Finland

Northern Ostrobothnia Hospital District, Oulu, Finland

Northern Ostrobothnia Hospital District, Oulu, Finland

Institute for Molecular Medicine Finland, HiLIFE, University of Helsinki, Finland Institute for Molecular Medicine Finland, HiLIFE, University of Helsinki, Finland Abbvie, Chicago, IL, United States

Biogen, Cambridge, MA, United States

Biogen, Cambridge, MA, United States

Genentech, San Francisco, CA, United States

Genentech, San Francisco, CA, United States

Genentech, San Francisco, CA, United States

Genentech, San Francisco, CA, United States

Northern Ostrobothnia Hospital District, Oulu, Finland Northern Ostrobothnia Hospital District, Oulu, Finland

Hospital District of Helsinki and Uusimaa, Helsinki, Finland

Pirkanmaa Hospital District, Tampere, Finland

Hospital District of Southwest Finland, Turku, Finland

Hospital District of Southwest Finland, Turku, Finland

Abbvie, Chicago, IL, United States

Abbvie, Chicago, IL, United States

Abbvie, Chicago, IL, United States

Genentech, San Francisco, CA, United States

Genentech, San Francisco, CA, United States

Janssen Research \& Development, LLC, Spring House, PA, United States

Pfizer, New York, NY, United States

Pfizer, New York, NY, United States

Hospital District of Helsinki and Uusimaa, Helsinki, Finland Hospital District of Helsinki and Uusimaa, Helsinki, Finland Hospital District of Helsinki and Uusimaa, Helsinki, Finland Hospital District of Helsinki and Uusimaa, Helsinki, Finland Hospital District of Helsinki and Uusimaa, Helsinki, Finland Hospital District of Helsinki and Uusimaa, Helsinki, Finland Hospital District of Helsinki and Uusimaa, Helsinki, Finland Northern Savo Hospital District, Kuopio, Finland Northern Savo Hospital District, Kuopio, Finland Northern Savo Hospital District, Kuopio, Finland Hospital District of Southwest Finland, Turku, Finland Northern Ostrobothnia Hospital District, Oulu, Finland Northern Ostrobothnia Hospital District, Oulu, Finland Genentech, San Francisco, CA, United States 


\section{Women's Health and Reproduction Group

Venla Kurra

Oskari Heikinheimo

926 Ilkka Kalliala
Kari Nieminen

Päivi Polo

Kaarin Mäkikallio

Eeva Ekholm

Marja Vääräsmäki

Outi Uimari

Laure Morin-Papunen

Marjo Tuppurainen

Katja Kivinen

Elisabeth Widen

Taru Tukiainen

Mary Pat Reeve

Mark Daly

Liu Aoxing

Andrea Ganna

Eija Laakkonen

Niko Välimäki

Lauri Aaltonen

Johannes Kettunen

Mikko Arvas

Jeffrey Waring

Bridget Riley-Gillis

Mengzhen Liu

Sarah Pendergrass

Janet Kumar

Kirsi Auro
Justin Wade Davis

Bridget Riley-Gillis

Reza Hammond

Fedik Rahimov

Sabah Kadri

Sahar Esmaeeli

Mengzhen Liu

Slavé Petrovski

Eleonor Wigmore

Adele Mitchell

Benjamin Sun

Ellen Tsai

Denis Baird

Paola Bronson

Ruoyu Tian

Stephanie Loomis

Yunfeng Huang

Joseph Maranville

Shameek Biswas

Elmutaz Mohammed

Samir Wadhawan

Erika Kvikstad

Minal Caliskan

Diana Chang

Julie Hunkapiller

Tushar Bhangale

Natalie Bowers

Sarah Pendergrass

Karen S King

Padhraig Gormley

Jimmy Liu

Karsten Sieber

Amy Hart
Pirkanmaa Hospital District, Tampere, Finland

Hospital District of Helsinki and Uusimaa, Helsinki, Finland

Hospital District of Helsinki and Uusimaa, Helsinki, Finland

Pirkanmaa Hospital District, Tampere, Finland

Pirkanmaa Hospital District, Tampere, Finland

Hospital District of Southwest Finland, Turku, Finland

Hospital District of Southwest Finland, Turku, Finland

Hospital District of Southwest Finland, Turku, Finland

Northern Ostrobothnia Hospital District, Oulu, Finland

Northern Ostrobothnia Hospital District, Oulu, Finland

Northern Ostrobothnia Hospital District, Oulu, Finland

Northern Savo Hospital District, Kuopio, Finland

Institute for Molecular Medicine Finland, HiLIFE, University of Helsinki, Finland Institute for Molecular Medicine Finland, HiLIFE, University of Helsinki, Finland Institute for Molecular Medicine Finland, HiLIFE, University of Helsinki, Finland Institute for Molecular Medicine Finland, HiLIFE, University of Helsinki, Finland Institute for Molecular Medicine Finland, HiLIFE, University of Helsinki, Finland Institute for Molecular Medicine Finland, HiLIFE, University of Helsinki, Finland Institute for Molecular Medicine Finland, HiLIFE, University of Helsinki, Finland University of Jyväskylä, Jyväskylä, Finland

University of Helsinki, Helsinki, Finland

Hospital District of Helsinki and Uusimaa, Helsinki, Finland

Northern Ostrobothnia Hospital District, Oulu, Finland

Finnish Red Cross Blood Service, Helsinki, Finland

Abbvie, Chicago, IL, United States

Abbvie, Chicago, IL, United States

Abbvie, Chicago, IL, United States

Genentech, San Francisco, CA, United States

GlaxoSmithKline, Brentford, United Kingdom

GlaxoSmithKline, Brentford, United Kingdom

FinnGen Analysis working group

Abbvie, Chicago, IL, United States

Abbvie, Chicago, IL, United States

Abbvie, Chicago, IL, United States

Abbvie, Chicago, IL, United States

Abbvie, Chicago, IL, United States

Abbvie, Chicago, IL, United States

Abbvie, Chicago, IL, United States

Astra Zeneca, Cambridge, United Kingdom

Astra Zeneca, Cambridge, United Kingdom

Biogen, Cambridge, MA, United States

Biogen, Cambridge, MA, United States

Biogen, Cambridge, MA, United States

Biogen, Cambridge, MA, United States

Biogen, Cambridge, MA, United States

Biogen, Cambridge, MA, United States

Biogen, Cambridge, MA, United States

Biogen, Cambridge, MA, United States

Celgene, Summit, NJ, United States/ Bristol Myers Squibb, New York, NY, United States Celgene, Summit, NJ, United States/ Bristol Myers Squibb, New York, NY, United States Celgene, Summit, NJ, United States/ Bristol Myers Squibb, New York, NY, United States Celgene, Summit, NJ, United States/ Bristol Myers Squibb, New York, NY, United States Celgene, Summit, NJ, United States/ Bristol Myers Squibb, New York, NY, United States Celgene, Summit, NJ, United States/ Bristol Myers Squibb, New York, NY, United States Genentech, San Francisco, CA, United States

Genentech, San Francisco, CA, United States Genentech, San Francisco, CA, United States Genentech, San Francisco, CA, United States Genentech, San Francisco, CA, United States GlaxoSmithKline, Brentford, United Kingdom GlaxoSmithKline, Brentford, United Kingdom GlaxoSmithKline, Brentford, United Kingdom Janssen Research \& Development, LLC, Spring House, PA, United States Janssen Research \& Development, LLC, Spring House, PA, United States 


\begin{tabular}{|c|c|c|}
\hline 989 & Meijian Guan & Janssen Research \& Development, LLC, Spring House, PA, United States \\
\hline 990 & Shicheng Guo & Janssen Research \& Development, LLC, Spring House, PA, United States \\
\hline 991 & Beryl Cummings & Maze Therapeutics, San Francisco, CA, United States \\
\hline 992 & Matt Brauer & Maze Therapeutics, San Francisco, CA, United States \\
\hline 993 & Jason Miller & Merck, Kenilworth, NJ, United States \\
\hline 994 & Fabiana Farias & Merck, Kenilworth, NJ, United States \\
\hline 995 & Jorge Del-Aguila & Merck, Kenilworth, NJ, United States \\
\hline 996 & Kirill Shkura & Merck, Kenilworth, NJ, United States \\
\hline 997 & Victor Neduva & Merck, Kenilworth, NJ, United States \\
\hline 998 & Huilei $\mathrm{Xu}$ & Novartis, Basel, Switzerland \\
\hline 999 & Amy Cole & Novartis, Basel, Switzerland \\
\hline 1000 & Jonathan Chung & Novartis, Basel, Switzerland \\
\hline 1001 & Jaison Jacob & Novartis, Basel, Switzerland \\
\hline 1002 & Katrina de Lange & Novartis, Basel, Switzerland \\
\hline 1003 & Jonas Zierer & Novartis, Basel, Switzerland \\
\hline 1004 & Xing Chen & Pfizer, New York, NY, United States \\
\hline 1005 & Åsa Hedman & Pfizer, New York, NY, United States \\
\hline 1006 & Clarence Wang & Sanofi, Paris, France \\
\hline 1007 & Ethan $\mathrm{Xu}$ & Sanofi, Paris, France \\
\hline 1008 & Franck Auge & Sanofi, Paris, France \\
\hline 1009 & Clement Chatelain & Sanofi, Paris, France \\
\hline 1010 & Deepak Rajpal & Sanofi, Paris, France \\
\hline 1011 & Dongyu Liu & Sanofi, Paris, France \\
\hline 1012 & Katherine Call & Sanofi, Paris, France \\
\hline 1013 & Tai-He Xia & Sanofi, Paris, France \\
\hline 1014 & Mitja Kurki & Institute for Molecular Medicine Finland, HiLIFE, University of Helsinki, Finland / Broad \\
\hline 1015 & \multicolumn{2}{|c|}{ Institute, Cambridge, MA, United States } \\
\hline 1016 & Samuli Ripatti & Institute for Molecular Medicine Finland, HiLIFE, University of Helsinki, Finland \\
\hline 1017 & Mark Daly & Institute for Molecular Medicine Finland, HiLIFE, University of Helsinki, Finland \\
\hline 1018 & Juha Karjalainen & Institute for Molecular Medicine Finland, HiLIFE, University of Helsinki, Finland \\
\hline 1019 & Aki Havulinna & Institute for Molecular Medicine Finland, HiLIFE, University of Helsinki, Finland \\
\hline 1020 & Juha Mehtonen & Institute for Molecular Medicine Finland, HiLIFE, University of Helsinki, Finland \\
\hline 1021 & Priit Palta & Institute for Molecular Medicine Finland, HiLIFE, University of Helsinki, Finland \\
\hline 1022 & Shabbeer Hassan & Institute for Molecular Medicine Finland, HiLIFE, University of Helsinki, Finland \\
\hline 1023 & Pietro Della Briotta Parolo & Institute for Molecular Medicine Finland, HiLIFE, University of Helsinki, Finland \\
\hline 1024 & Wei Zhou & Broad Institute, Cambridge, MA, United States \\
\hline 1025 & Mutaamba Maasha & Broad Institute, Cambridge, MA, United States \\
\hline 1026 & Shabbeer Hassan & Institute for Molecular Medicine Finland, HiLIFE, University of Helsinki, Finland \\
\hline 1027 & Susanna Lemmelä & Institute for Molecular Medicine Finland, HiLIFE, University of Helsinki, Finland \\
\hline 1028 & Manuel Rivas & University of Stanford, Stanford, CA, United States \\
\hline 1029 & Aarno Palotie & Institute for Molecular Medicine Finland, HiLIFE, University of Helsinki, Finland \\
\hline 1030 & Arto Lehisto & Institute for Molecular Medicine Finland, HiLIFE, University of Helsinki, Finland \\
\hline 1031 & Andrea Ganna & Institute for Molecular Medicine Finland, HiLIFE, University of Helsinki, Finland \\
\hline 1032 & Vincent Llorens & Institute for Molecular Medicine Finland, HiLIFE, University of Helsinki, Finland \\
\hline 1033 & Hannele Laivuori & Institute for Molecular Medicine Finland, HiLIFE, University of Helsinki, Finland \\
\hline 1034 & Mari E Niemi & Institute for Molecular Medicine Finland, HiLIFE, University of Helsinki, Finland \\
\hline 1035 & Taru Tukiainen & Institute for Molecular Medicine Finland, HiLIFE, University of Helsinki, Finland \\
\hline 1036 & Mary Pat Reeve & Institute for Molecular Medicine Finland, HiLIFE, University of Helsinki, Finland \\
\hline 1037 & Henrike Heyne & Institute for Molecular Medicine Finland, HiLIFE, University of Helsinki, Finland \\
\hline 1038 & Nina Mars & Institute for Molecular Medicine Finland, HiLIFE, University of Helsinki, Finland \\
\hline 1039 & Kimmo Palin & University of Helsinki, Helsinki, Finland \\
\hline 1040 & Javier Garcia-Tabuenca & University of Tampere, Tampere, Finland \\
\hline 1041 & Harri Siirtola & University of Tampere, Tampere, Finland \\
\hline 1042 & Tuomo Kiiskinen & Institute for Molecular Medicine Finland, HiLIFE, University of Helsinki, Finland \\
\hline 1043 & Jiwoo Lee & Institute for Molecular Medicine Finland, HiLIFE, University of Helsinki, Finland / Broad \\
\hline 1044 & \multicolumn{2}{|c|}{ Institute, Cambridge, MA, United States } \\
\hline 1045 & Kristin Tsuo & Institute for Molecular Medicine Finland, HiLIFE, University of Helsinki, Finland / Broad \\
\hline 1046 & \multicolumn{2}{|c|}{ Institute, Cambridge, MA, United States } \\
\hline 1047 & Amanda Elliott & Institute for Molecular Medicine Finland, HiLIFE, University of Helsinki, Finland / Broad \\
\hline 1048 & \multicolumn{2}{|c|}{ Institute, Cambridge, MA, United States } \\
\hline 1049 & Kati Kristiansson & THL Biobank / The National Institute of Health and Welfare Helsinki, Finland \\
\hline 1050 & Mikko Arvas & Finnish Red Cross Blood Service / Finnish Hematology Registry and Clinical Biobank, \\
\hline 1051 & Helsinki, Finland & \\
\hline 1052 & Kati Hyvärinen & Finnish Red Cross Blood Service, Helsinki, Finland \\
\hline 1053 & Jarmo Ritari & Finnish Red Cross Blood Service, Helsinki, Finland \\
\hline 1054 & Miika Koskinen & Helsinki Biobank / Helsinki University and Hospital District of Helsinki and Uusimaa, \\
\hline
\end{tabular}

1055

Helsinki 
1056

1057

1058

1059

1060

1061

1062

1063

1064

1065

1066

1067

1068

1069

1070

1071

1072

1073

1074

1075

1076

1077

1078

1079

1080

1081

1082

1083

1084

1085

1086

1087

1088

1089

1090

1091

1092

1093

1094

1095

1096

1097

1098

1099

1100

1101

1102

1103

1104

1105

1106

1107

1108

1109

1110

1111

1112

1113

1114

1115

1116

1117

1118

1119

1120

1121

1122
Helsinki Biobank / Helsinki University and Hospital District of Helsinki and Uusimaa,

Northern Finland Biobank Borealis / University of Oulu / Northern Ostrobothnia Hospital

Canki

Johannes Kettunen

District, Oulu, Finland

Katri Pylkäs

Eeva Sliz

Minna Karjalainen

Tuomo Mantere

District, Oulu, Finland

Eeva Kangasniemi

Tampere, Finland

Sami Heikkinen

Arto Mannermaa

Kuopio, Finland

Eija Laakkonen

Dhanaprakash Jambulingam University of Turku, Turku, Finland

Venkat Subramaniam Rathinakannan University of Turku, Turku, Finland

Nina Pitkänen

Auria Biobank / University of Turku / Hospital District of Southwest Finland, Turku, Finland

Biobank directors

Lila Kallio

Sirpa Soini

Jukka Partanen

Helsinki, Finland

Eero Punkka

Helsinki

Raisa Serpi

District, Oulu, Finland

Kimmo Savinainen (acting) Finnish Clinical Biobank Tampere / University of Tampere / Pirkanmaa Hospital District, Tampere, Finland

Veli-Matti Kosma

Kuopio, Finland

Teijo Kuopio

Jyväskylä, Finland

Auria Biobank / University of Turku / Hospital District of Southwest Finland, Turku, Finland THL Biobank / The National Institute of Health and Welfare Helsinki, Finland

Finnish Red Cross Blood Service / Finnish Hematology Registry and Clinical Biobank,

Helsinki Biobank / Helsinki University and Hospital District of Helsinki and Uusimaa,

Northern Finland Biobank Borealis / University of Oulu / Northern Ostrobothnia Hospital Biobank of Eastern Finland / University of Eastern Finland / Northern Savo Hospital District,

Central Finland Biobank / University of Jyväskylä / Central Finland Health Care District,

FinnGen Teams

Administration

Anu Jalanko

Huei-Yi Shen

Risto Kajanne

Mervi Aavikko

Institute for Molecular Medicine Finland, HiLIFE, University of Helsinki, Finland Institute for Molecular Medicine Finland, HiLIFE, University of Helsinki, Finland Institute for Molecular Medicine Finland, HiLIFE, University of Helsinki, Finland Institute for Molecular Medicine Finland, HiLIFE, University of Helsinki, Finland

Analysis

Mitja Kurki

Institute for Molecular Medicine Finland, HiLIFE, University of Helsinki, Finland / Broad Institute, Cambridge, MA, United States

Juha Karjalainen

Institute for Molecular Medicine Finland, HiLIFE, University of Helsinki, Finland

Pietro Della Briotta Parolo Institute for Molecular Medicine Finland, HiLIFE, University of Helsinki, Finland

Arto Lehisto

Juha Mehtonen

Wei Zhou

Masahiro Kanai

Institute for Molecular Medicine Finland, HiLIFE, University of Helsinki, Finland Institute for Molecular Medicine Finland, HiLIFE, University of Helsinki, Finland

Broad Institute, Cambridge, MA, United States

Broad Institute, Cambridge, MA, United States

Mutaamba Maasha

Broad Institute, Cambridge, MA, United States

\section{Clinical Endpoint Development}

Hannele Laivuori

Aki Havulinna

Susanna Lemmelä

Tuomo Kiiskinen

L. Elisa Lahtela

Institute for Molecular Medicine Finland, HiLIFE, University of Helsinki, Finland Institute for Molecular Medicine Finland, HiLIFE, University of Helsinki, Finland Institute for Molecular Medicine Finland, HiLIFE, University of Helsinki, Finland Institute for Molecular Medicine Finland, HiLIFE, University of Helsinki, Finland Institute for Molecular Medicine Finland, HiLIFE, University of Helsinki, Finland

\section{Communication}

Mari Kaunisto

Institute for Molecular Medicine Finland, HiLIFE, University of Helsinki, Finland

\section{E-Science}

Elina Kilpeläinen

Timo P. Sipilä

Institute for Molecular Medicine Finland, HiLIFE, University of Helsinki, Finland Institute for Molecular Medicine Finland, HiLIFE, University of Helsinki, Finland Oluwaseun Alexander Dada Institute for Molecular Medicine Finland, HiLIFE, University of Helsinki, Finland 
medRxiv preprint doi: https://doi.org/10.1101/2021.08.20.21262098; this version posted August 25, 2021. The copyright holder for this preprint (which was not certified by peer review) is the author/funder, who has granted medRxiv a license to display the preprint in perpetuity. It is made available under a CC-BY-ND 4.0 International license .

1123 Awaisa Ghazal

1124 Anastasia Shcherban

1125

1126

1127

1128

1129

1130

1131

1132

1133

1134

1135

1136

1137

1138

1139

1140

1141

1142

1143

1144

1145

1146

1147

1148

1149

1150

1151

1152

1153

1154

1155

1156

1157

1158

1159

1160

1161

1162

1163

1164

1165

1166

1167

1168

1169

1170

1171

1172

1173

1174

1175

1176

1177

\section{Genotyping}

Kati Donner

Timo P. Sipilä

Anu Loukola

Helsinki

\section{Sample Logistics \\ Päivi Laiho \\ Tuuli Sistonen \\ Essi Kaiharju \\ Markku Laukkanen \\ Elina Järvensivu \\ Sini Lähteenmäki \\ Lotta Männikkö \\ Regis Wong}

Hannele Mattsson

Kati Kristiansson

Susanna Lemmelä

Tero Hiekkalinna

Teemu Paajanen

Priit Palta

Kalle Pärn

Mart Kals

Shuang Luo

Vishal Sinha

\section{Trajectory}

Mary Pat Reeve

Marianna Niemi

Harri Siirtola

Mika Helminen

Tiina Luukkaala

Iida Vähätalo

Tero Jyrhämä

Marco Hautalahti

Johanna Mäkelä

Laura Mustaniemi

Mirkka Koivusalo

Sarah Smith

Tom Southerington
Institute for Molecular Medicine Finland, HiLIFE, University of Helsinki, Finland

Institute for Molecular Medicine Finland, HiLIFE, University of Helsinki, Finland

Sample Collection Coordination

Institute for Molecular Medicine Finland, HiLIFE, University of Helsinki, Finland

Institute for Molecular Medicine Finland, HiLIFE, University of Helsinki, Finland

Helsinki Biobank / Helsinki University and Hospital District of Helsinki and Uusimaa,

Registry Data Operations

Sami Koskelainen

\section{Sequencing Informatics}

Tarja Laitinen

Javier Gracia-Tabuenca

\section{Data protection officer}

\section{FINBB - Finnish biobank cooperative}

THL Biobank / The National Institute of Health and Welfare Helsinki, Finland THL Biobank / The National Institute of Health and Welfare Helsinki, Finland THL Biobank / The National Institute of Health and Welfare Helsinki, Finland THL Biobank / The National Institute of Health and Welfare Helsinki, Finland THL Biobank / The National Institute of Health and Welfare Helsinki, Finland THL Biobank / The National Institute of Health and Welfare Helsinki, Finland THL Biobank / The National Institute of Health and Welfare Helsinki, Finland THL Biobank / The National Institute of Health and Welfare Helsinki, Finland

THL Biobank / The National Institute of Health and Welfare Helsinki, Finland THL Biobank / The National Institute of Health and Welfare Helsinki, Finland Institute for Molecular Medicine Finland, HiLIFE, University of Helsinki, Finland THL Biobank / The National Institute of Health and Welfare Helsinki, Finland THL Biobank / The National Institute of Health and Welfare Helsinki, Finland THL Biobank / The National Institute of Health and Welfare Helsinki, Finland

Institute for Molecular Medicine Finland, HiLIFE, University of Helsinki, Finland Institute for Molecular Medicine Finland, HiLIFE, University of Helsinki, Finland Institute for Molecular Medicine Finland, HiLIFE, University of Helsinki, Finland Institute for Molecular Medicine Finland, HiLIFE, University of Helsinki, Finland Institute for Molecular Medicine Finland, HiLIFE, University of Helsinki, Finland

Pirkanmaa Hospital District, Tampere, Finland Institute for Molecular Medicine Finland, HiLIFE, University of Helsinki, Finland University of Tampere, Tampere, Finland University of Tampere, Tampere, Finland University of Tampere, Tampere, Finland University of Tampere, Tampere, Finland University of Tampere, Tampere, Finland University of Tampere, Tampere, Finland

Institute for Molecular Medicine Finland, HiLIFE, University of Helsinki, Finland 